\title{
An automatic road sign recognition system based on a computational model of human recognition processing
}

\author{
C.Y. Fang ${ }^{\mathrm{a}, *}$, C.S. Fuh ${ }^{\mathrm{b}}$, P.S. Yen ${ }^{\mathrm{a}}$, S. Cherng ${ }^{\mathrm{c}}$, S.W. Chen ${ }^{\mathrm{d}}$ \\ a Department of Information and Computer Education, National Taiwan Normal University, \\ Taipei, Taiwan, ROC \\ ${ }^{\mathrm{b}}$ Department of Computer Science and Information Engineering, National Taiwan University, \\ Taipei, Taiwan, $R O C$ \\ ${ }^{\mathrm{c}}$ Department of Electrical Engineering, Cheng Shiu University, Kaohsiung, Taiwan, ROC \\ d Department of Computer Science and Information Engineering, National Taiwan Normal University, \\ Taipei, Taiwan, $R O C$
}

Received 14 March 2002; accepted 2 February 2004

Available online 3 August 2004

\begin{abstract}
This paper presents an automatic road sign detection and recognition system that is based on a computational model of human visual recognition processing. Road signs are typically placed either by the roadside or above roads. They provide important information for guiding, warning, or regulating the behaviors drivers in order to make driving safer and easier. The proposed recognition system is motivated by human recognition processing. The system consists of three major components: sensory, perceptual, and conceptual analyzers. The sensory analyzer extracts the spatial and temporal information of interest from video sequences. The extracted information then serves as the input stimuli to a spatiotemporal attentional (STA) neural network in the perceptual analyzer. If stimulation continues, focuses of attention will be established in the neural network. Potential features of road signs are then extracted from the image areas corresponding to the focuses of attention. The extracted features are next fed into the conceptual analyzer. The conceptual analyzer is composed of two modules: a category module and an object module. The former uses a configurable adaptive resonance
\end{abstract}

\footnotetext{
${ }^{*}$ Corresponding author. Fax: +886-2-23512772.

E-mail address: violet@ice.ntnu.edu.tw (C.Y. Fang).
} 
theory (CART) neural network to determine the category of the input stimuli, whereas the later uses a configurable heteroassociative memory (CHAM) neural network to recognize an object in the determined category of objects. The proposed computational model has been used to develop a system for automatically detecting and recognizing road signs from sequences of traffic images. The experimental results revealed both the feasibility of the proposed computational model and the robustness of the developed road sign detection system.

(c) 2004 Elsevier Inc. All rights reserved.

Keywords: Road sign recognition; Cognitive model; Sensory, perceptual, and conceptual analyzer; Spatiotemporal attentional neural network; Configurable adaptive resonance theory neural network; Configurable associative memory neural network

\section{Introduction}

\subsection{Motivation}

The main purpose of driving assistance systems (DAS) is to collect significant information for drivers in order to reduce their effort in safe driving. Drivers have to pay attention to various conditions, including vehicle speed and orientation, the distance between vehicles, passing cars, and potential dangerous or unusual events ahead. If DAS can collect such information a prior, it will greatly reduce the burden of driving for drivers and make driving safer and easier.

Driving information mentioned above could be detected by many kinds of devices, such as infrared rays, ultrasonic waves, microwaves, radar, and a computer vision system. These devices can be utilized to extract various kinds of data from the driving environments. Computer vision systems, which have the advantage of high-resolution, can be employed to detect road borders and obstacles, and recognize road signs. This kind of device cannot be surpassed because only they can "see" and "recognize" something during driving without the use extra auxiliary devices such as magnetic marks.

Road signs are installed to guide, warn, and regulate traffic. They supply information to help drivers operate their cars in such a way as to ensure traffic safety. In the real world, when they get tired, drivers may not always notice road signs. At night, drivers are easily affected by headlights of on coming vehicles and may miss road signs. In bad weather, road signs are harder to recognize quickly and correctly. These situations may lead to traffic accidents and serious injuries. A vision-based road sign detection and recognition system is thus desirable to catch the attention of a driver to avoid traffic hazards.

Road signs are designed to attract a driver's attention with particular colors and simple geometric shapes. However, the difficulty in recognizing road signs is largely due to the following reasons:

(1) The colors of road signs, particularly red, may fade after long exposure to the sun. Moreover, paint may even flake or peel off. 
(2) Air pollution and weather conditions (e.g., rain, fog, shadows, and clouds) may decrease the visibility of road signs.

(3) Outdoor lighting conditions varying from day to night may affect the colors of road signs.

(4) Obstacles, such as vehicles, pedestrians, and other road signs, may partially occlude road signs.

(5) Video images of road signs will have motion blur if the camcorder is mounted on a moving vehicle due to vehicle vibration as well as motion.

\subsection{Related work}

Vision systems incorporating visual sensing devices have been used in a variety of applications, such as recognition, classification, detection, tracking, and monitoring. For the purpose of driver assistance, vision systems have been employed to detect, recognize, and track objects such as lanes [3,22], roads [5,15,20,24], road signs and traffic signals [6,30], road conditions (e.g., dry, wet, ice, and snow) [35], obstacles (e.g., pedestrians, bikes, motorcycles, vehicles, and other intruders) [3-5,18,28], as well as structures (e.g., tunnels, bridges, and overpasses) [11].

Since no formal definition for a vision-based driver assistance system (VDAS) has ever been given, any functions, as mentioned above, that contribute to driving safety can be considered a part of VDAS. To realize these functions, we have proposed a dynamic visual model (DVM), which is a computational model for visual analysis based on video sequences. The model primarily inspired by the human visual system provides an underlying framework in which road sign detection system is developed. Our DVM bridges some techniques of computer vision and some properties of human vision. In this study, we design artificial systems with similar properties of natural systems and hope to improve the understanding of natural process through the operations of artificial systems. Note that the proposed DVM is a general-purpose model. Various visual application systems using video sequences as input data will be developed on the basis of the proposed model.

Recently, many techniques have been developed to detect and recognize road signs. Pacheco et al. [29] proposed adding special color barcodes under road signs to help road sign identification for vision-based systems. However, much time resources would be expended to reequip road signs, making this solution not economical. Aoyagi and Asakura [1] used genetic algorithms to detection road signs from gray-level video imagery. Unfortunately, because of the limitation of crossover and mutation operators, optimal solutions are not guaranteed. Lalonde and $\mathrm{Li}$ [23] reported a color indexing approach to identify road signs, but the computation time increases greatly in complex traffic scenes. Additionally, many other studies about detecting and recognizing road signs by distance transforms [13], morphological methods [17], neural networks [14,16,19,25], and fuzzy reasoning [24] have been presented.

Researchers attempt to detect and recognize road signs not only in a single image $[8,9,19,31,36]$, but also in a video sequence $[2,7,30,32]$. Practically, at any given 
moment road signs may be partially occluded by vehicles, pedestrians, or other obstacles. Using video sequences, we could preserve useful information from earlier image frames, such as the number and sizes of road signs, to predict sizes and positions of road signs in later frames. This information will increase the speed of road sign detection and the correctness of road sign recognition in later frames. At the same time, information supplied by the later frames will help with road sign verification for earlier frames. In summary, video sequences provide much more useful information than single images.

Road signs are always placed either beside roads or above lanes. Being intended to guide, warn, or regulate traffic to make driving safer and easier, they are designed to attract drivers' attention to provide important information. It is believed that if the behavior of an automatic road sign recognition system can be patterned after the human brain, then such a system could ultimately detect and recognize road signs from video image sequences as correctly and quickly as people do. This paper describes an automatic road sign detection and recognition system based on a computational model of human recognition processing. We introduce in Section 2 a computational model for pattern recognition inspired by human cognitive processing. There are three important neural networks incorporated in the computational model: spatiotemporal attentional (STA), configurable adaptive resonance theory (CART), and configurable heteroassociative memory (CHAM). A system based on the proposed computational model for detecting and recognizing road signs is developed in Section 3. The feasibility of the proposed computational model and the robustness of the developed road sign detection and recognition system are explored in Section 4. Finally, we draw conclusions and address future work in Section 5.

\section{Computational model}

\subsection{Psychological fundamentals}

While computers are excellent at computing, they are unhandy in both object detection and pattern recognition. On the other hand, the human brain exhibits impressive powers in detection and recognition, but are not as good as computers in computational ability. Human detection and recognition is primarily characterized by two features: parallel processing and distributed representation. In the distributed representation of information, a subject matter is decomposed into simple concepts, each of which is encoded by a cognitive unit (a single neuron or a small set of neurons collectively acting as a complex neuron). Furthermore, since a concept may be shared by several different subjects, a cognitive unit can be involved in representing many subjects.

In parallel processing, cognitive units can simultaneously process their own received stimuli and propagate their outputs to others. Information, which is scattered everywhere in the cortex, can be interchanged immediately and in parallel through the connections among cognitive units. By this exchange process, high-level information, including abstract concepts, can be constructed and supplied to the brain to 
analyze, thereby allowing humans to understand complex situations, including those which cannot be worked out by a sequential process. For this reason we designed a computational framework to model the recognition process of the human brain for object detection and pattern recognition (see Fig. 1).

Before discussing our computational model, we give a brief description of human cognition and recognition. According to cognitive psychologists [26], there are hierarchical levels of information processing involved in cognition and recognition, such as sensory, perceptual, conceptual, and action levels, as shown in Fig. 2. Considering the case of vision, light (physical stimuli) absorbed by rods and cones (receptors) is transformed into electrical signals, which are transmitted to the visual sensation analyzer (the sensory analyzer) located in the primary visual receiving areas of the cortex. The visual sensation analyzer extracts basic features (e.g., edges, lines, and corners), spatial frequency patterns, as well as the information about color, depth, and motion from the input signals. The outputs of the visual sensation analyzer are delivered to the visual analyzer (the perceptual analyzer). The visual analyzer lying in the visual association areas of the cortex perceives the categories of the input stimuli (e.g., objects) based on the features detected at the sensory level. Both the sensed low-level features and the perceived high-level categories are exploited at the conceptual level to understand the input stimuli. Finally, people take action if necessary, and the motor system takes care of the details of muscle movements.

All analyzers involved in cognition and recognition have analogous architectures [21]. Every analyzer contains several layers of neurons, called cognitive units. Cognitive units on the same layer are laterally connected with links which are almost always inhibitory. Cognitive units on different layers are vertically connected with links which are excitatory. A vertical link indicates the existence of a particular part-whole relationship; the synaptic strength of the link specifies the degree of the relationship. A lateral inhibitory link signifies the existence of a mutually exclusive relationship between two components; the strength of the link describes the grade of the relationship. Both links and their strengths are made up solely through learning.

In the above, the parallel processing, distributed representation, hierarchical analyzers, and layered architecture of the human brain are discussed. Numerous

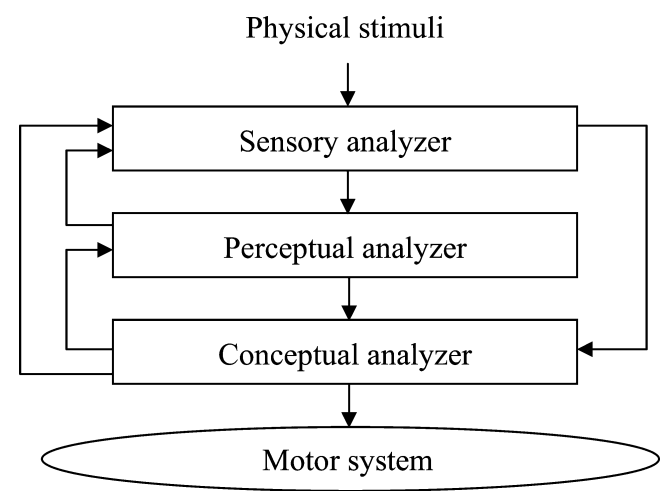

Fig. 1. A simplified version of human cognitive and recognition processing. 


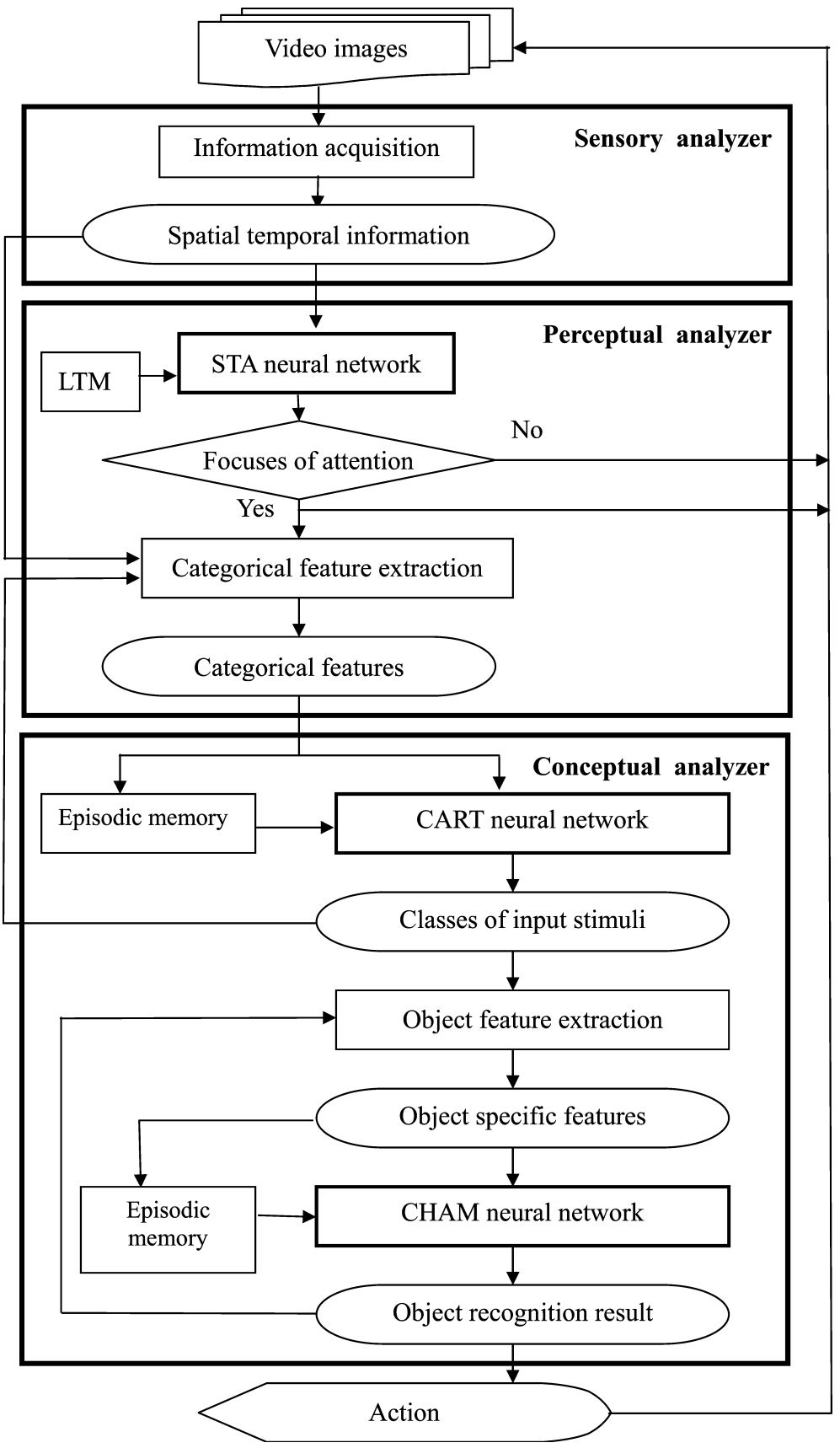

Fig. 2. The proposed computational model. 
functions of the brain are brought into being on the basis of the above features. In Section 2.2, a computational model motivated by human cognitive processing for road sign detection and recognition is developed.

\subsection{Cognitive model}

Fig. 2 depicts the proposed computational model. There are three analyzers comprising of the model, referred to as sensory, perceptual, and conceptual. The input data to the model are video sequences, which carry both spatial and temporal information. Spatial information sketches the relations (e.g., distance, direction) between objects in a single image, and temporal information describes the change of objects (e.g., color, intensity, and position) between successive images. All these kinds of information are important for correct detection and recognition. First, in the sensory analyzer, we extract the temporal and spatial information of moving objects from the input video sequence.

While a vehicle is in motion, everything outside the vehicle appears to be moving. How can the objects of interest be pulled from a jumble of moving objects? Intuitively, specific characteristics (regarding shape, color, and texture) of objects can be utilized to distinguish them. In the perceptual analyzer, a voluntary selectivity of attention is realized by introducing a network called the STA neural network, as well as a long-term memory (LTM), which preserves the characteristics of the objects of interest. The information from the LTM will call the attention of the neural network to the objects of interest when it is being innervated by the stimuli (i.e., the spatial and temporal information) coming from the sensory analyzer. Then the activations of the STA neurons are examined. If there is no focus of attention formed over the neurons, the system repeats the above process. Otherwise, the feature extraction step is evoked to detect categorical features of the objects within the image areas corresponding to the focuses of attention in the STA neural network.

Human brains first take notice of attractional objects, such as moving objects or brightly colored objects, and then recognize them from coarse to fine. For example, when we are driving, road signs become noticeable to us from afar. We may first distinguish what categories they belong to, such as prohibition signs or guide signs by their color or shape. Later, when the road signs appear larger, we can recognize their meaning, such as no right turn, no left turn, and so on. In our computational model, the classification stage, category perception, is simulated by CART neural network. The recognition stage, object recognition, is implemented by a CHAM neural network.

The categorical features obtained in the perceptual analyzer serve as the input stimuli, represented as a supraliminal pattern, to a CART neural network in the conceptual analyzer. Fig. 3 represents the flowchart of a CART neural network. The input supraliminal pattern first initializes the LTM of the CART neural network with the contents coming from a system memory, called the episodic memory. The configurations of the LTM and the associated components of the neural network have to be adapted in accordance with the contents. This adaptability of configuration is referred as the configurable capability of the neural network. Subliminal patterns to 


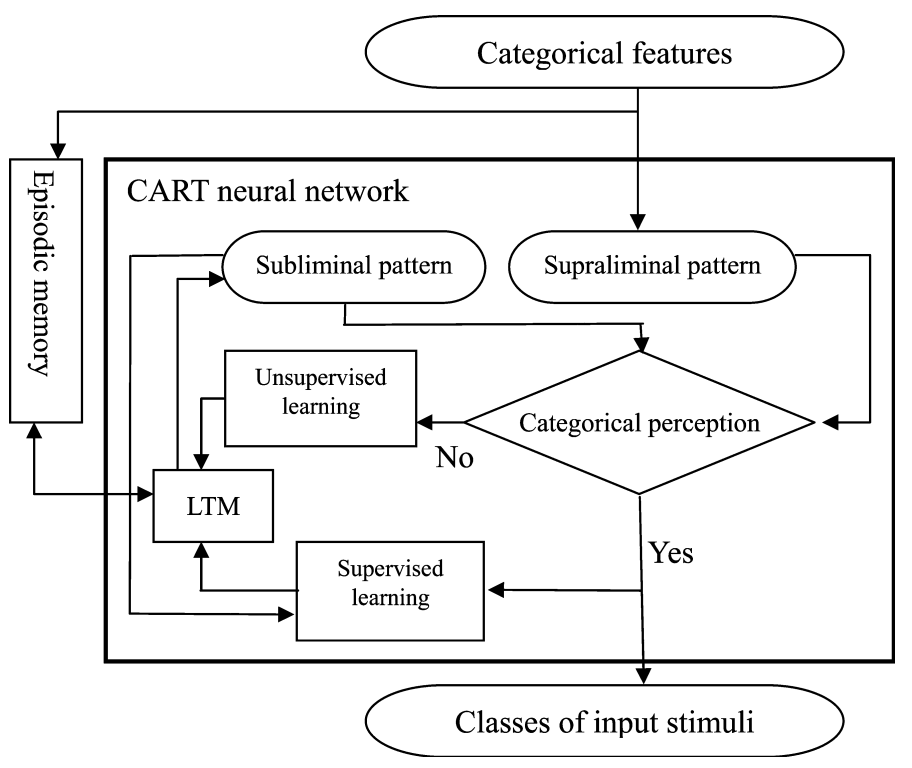

Fig. 3. Flowchart for CART neural network.

be matched with the input supraliminal pattern will be retrieved from the LTM, for which the search space of subliminal patterns is greatly reduced. The supraliminal pattern is compared with a subliminal pattern, and if they are similar enough, the class of the supraliminal pattern is regarded as that of the subliminal pattern under consideration. The CART network then performs a supervised learning through which the subliminal pattern in the LTM is updated under the guidance of the input supraliminal pattern. On the other hand, if no subliminal pattern is similar to any supraliminal pattern, an unsupervised learning, which represents the supraliminal pattern as a new subliminal pattern in the LTM, is carried out.

After the classification stage, particular object features regarding the special category are extracted and fed into a CHAM neural network, which is the recognition stage in the conceptual analyzer. Similar to the classification stage, the supraliminal object feature pattern first initializes the LTM of the CHAM network with the contents coming from the episodic memory. If the supraliminal pattern adequately matches a subliminal pattern, the supraliminal pattern is recognized successfully. Otherwise, our system is in a new situation and will attempt to learn and memorize the new experience for future recognition.

\section{Road sign detection and recognition system}

A system rooted in the proposed computational model for detecting and recognizing road signs is presented in this section. The system consists of three components: 
sensory, perceptual, and conceptual, corresponding to the three analyzers of the proposed computational model.

\subsection{Sensory component}

The input data to our system were acquired using a video camcorder (SONY TRV-900) mounted in the front windshield of a vehicle while driving on freeways. Since the vehicle's speed is high (more then $90 \mathrm{~km} / \mathrm{h}$ ), we use the interlace mode of SONY TRV 900 to capture video sequences. In this mode the images are clearer than in the normal mode and especially useful for analyzing high-speed actions such as traffic scenes. However, moving objects appear jerky if the image is read/output about every $1 / 30$ s.

In the sensory analyzer of our system, the spatial and temporal information of dynamic scenes is extracted from the input video sequences, and noise is filtered out. The sensory analyzer is a primary analyzer which concerns itself with only local information. In road sign detection, color is a local feature which can be extracted from a single pixel. On the other hand, shape is global feature and must be decided by a neighborhood of pixels.

As mentioned above, road sign detection is very difficult under poor weather conditions because of the influence of constantly varying outdoor illumination. Even though the actual colors of road signs are initially quite well controlled, the perceived colors are affected by illumination from light of various colors in their natural settings. Moreover, with the effects of sunlight, the paint on signs often fades with time. The hue component in HSI (hue, saturation, and intensity) model is invariant to brightness [33] and shadows. Thus, the hue component is suitable to extract color features, given the uncertainty of weather and natural and artificial damage to road signs.

There are one-to-one mappings of sensory analyzers to the pixels of an input image, and a sensory analyzer processes only the information coming from a single pixel. First, the hue value, $h$, of each pixel is calculated by

$$
h= \begin{cases}\frac{180}{\pi} \cos ^{-1}\left\{\frac{\frac{1}{2}[(r-g)+(r-b)]}{\left[(r-g)^{2}+(r-b)(g-b)\right]^{1 / 2}}\right\} & \text { if }(g-b) \geqslant 0, \\ -\frac{180}{\pi} \cos ^{-1}\left\{\frac{\frac{1}{2}[(r-g)+(r-b)]}{\left[(r-g)^{2}+(r-b)(g-b)\right]^{1 / 2}}\right\} & \text { if }(g-b)<0,\end{cases}
$$

where $r, g$, and $b$ indicate the red, green, and blue values of the pixel. Then, the similarity between $h$ and the stored hue values of particular colors in road signs is calculated. Let $\left\{h_{1}, h_{2}, \ldots, h_{q}\right\}$ be the set all the hue values of particular colors in road signs which are assumed to be Gaussianly distributed with variance $\sigma^{2}$. Then the output of the sensory analyzer is the degree of similarity

$$
z=\max _{k=1, \ldots, q}\left(z_{k}\right),
$$

where $z_{k}=\frac{1}{\sqrt{2 \pi} \sigma} \exp \left(\left(h-h_{k}\right)^{2} / 2 \sigma^{2}\right)$.

Finally, the outputs of sensory analyzers are fed into the perceptual analyzer. Fig. 4 gives an example showing the result of the sensory analyzers. The input image 
$\mathbf{A}$

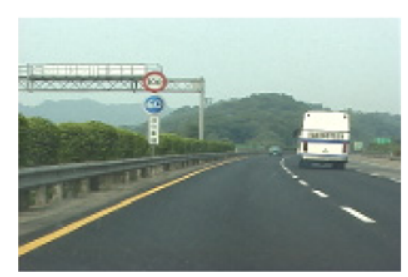

B

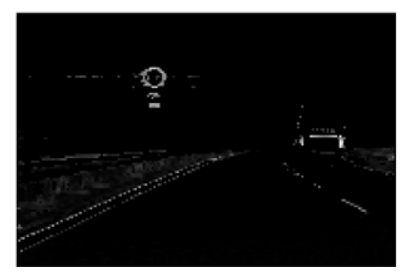

C

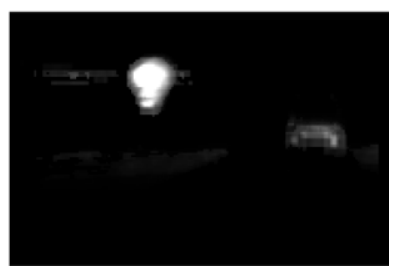

Fig. 4. An example of the attention map of the STA neural network. (A) One image of an input video sequence. (B) Corresponding outputs of the sensory analyzers. (C) Corresponding attention map of the STA neural network.

is shown in Fig. 4A. There are two road signs in the traffic scenes: one red and one blue. The output of the sensory analyzers is shown in Fig. 4B, and the intensities of the pixels indicate their degrees belonging to road sign colors.

\subsection{Perceptual component}

There are two major tasks in the perceptual component: attention formation and feature extraction. The former is finished by a STA neural network, and the latter by a categorical feature extraction method. These two techniques are introduced in Sections 3.2.1 and 3.2.2.

\subsubsection{STA neural network}

Tagare et al. [34] presented a maximum-likelihood strategy to direct the attention during visual search. This "attention strategy" can reduce the overall computation by suggesting a promising subregion of the image in which a target object may appear. The subregion is similar to the focus of attention in which humans are interested. However, their method works on static images. In our application, input video sequences are continuous stimuli carrying both spatial and temporal information of dynamic scenes. The STA neural network is proposed for processing spatial and temporal information in one construct.

We give only a brief description of the STA neural network in this subsection; more details can be found in [10]. The STA neural network, as Fig. 5 shows, is structured as a two-layer network: one for input and one for output. The output layer is also referred to as the attentional layer. Neurons in this layer are arranged into a 2D array in which they are connected to one another. These connections are within-layer (lateral) connections and are almost always inhibitory. There are no synaptic links among input neurons; they are, however, fully connected to the attentional neurons. These connections are called between-layer (vertical) connections and are always excitatory.

The input neurons are also organized into a $2 \mathrm{D}$ array as are the attentional neurons, and the size of both arrays are the same as that of the input images. Let $w_{i j}$ denote the weight of the link between attentional neuron $n_{i}$ and input neuron $n_{j}$. The weight vector of attentional neuron $n_{i}$ is written as $\mathbf{w}_{i}=\left(w_{i 1}, w_{i 2}, \ldots, w_{i m}\right)$, where $m$ 


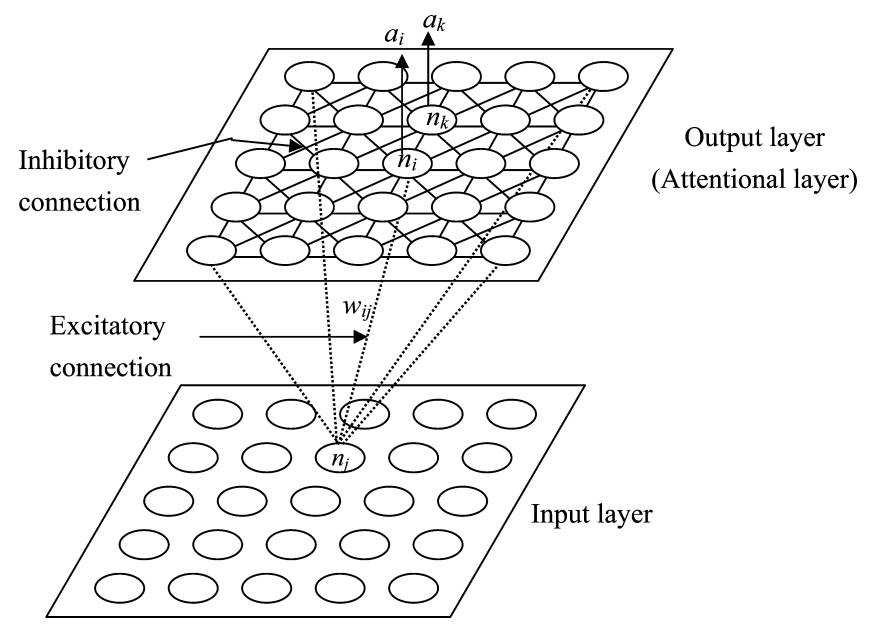

Fig. 5. STA neural network.

is the number of input neurons. The activation of attentional neuron $n_{i}$ due to input stimuli $\mathbf{z}$ (coming from sensory analyzers) is

$$
\begin{aligned}
& a_{i}(t)=\psi\left(a_{i}(t-1)+B\left(-\alpha a_{i}(t-1)+\beta A\left(I_{i}^{v}+I_{i}^{l}-\Gamma_{n}\right)\right)\right), \\
& I_{i}^{v}=\sum_{j=1}^{m} w_{i j} z_{j}, \quad I_{i}^{l}=\sum_{k \in N_{i}, k \neq i}\left[u_{i k} M\left(r_{i k}\right) a_{k}(t-1)\right], \\
& A(v)= \begin{cases}v & \text { if } v>0 \\
0 & \text { if } v \leqslant 0,\end{cases} \\
& B(v)=\left\{\begin{array}{ll}
v & \text { if } v>0 \\
\gamma v & \text { if } v \leqslant 0
\end{array}, \text { where } 1>\gamma>0 .\right.
\end{aligned}
$$

In the above equations, $a_{k}$ is the activation of neuron $n_{k}$, threshold $\Gamma_{n}$ prevents the effect due to noise, and $\alpha$ and $\beta$ are positive parameters. Set $N_{i}$ indicates the neighboring set of attentional neuron $n_{i} ; u_{i k}$ is the linking weight between neurons $n_{i}$ and $n_{k} ; M\left(\mathbf{r}_{i k}\right)$ denotes a "Mexican-hat" function, and the parameter $\mathbf{r}_{i k}$ is the distance between neurons $n_{i}$ and $n_{k}$.

The linking weights, $w_{i j}$, between the input and attentional layers are defined as follows. Referring to Fig. 6, let $n_{j}$ be any input neuron and $n_{i}$ be its corresponding attentional neuron. Assume that a 2D Gaussian $G$ is centered at attentional neuron $n_{i}$. Then, the linking strength $w_{k j}$ between input neuron $n_{j}$ and attentional neuron $n_{k}$ is defined as $w_{k j}=G\left(\mathbf{r}_{k i}\right)$, where $\mathbf{r}_{k i}$ is the position vector of neuron $n_{k}$ with respect to neuron $n_{i}$. 


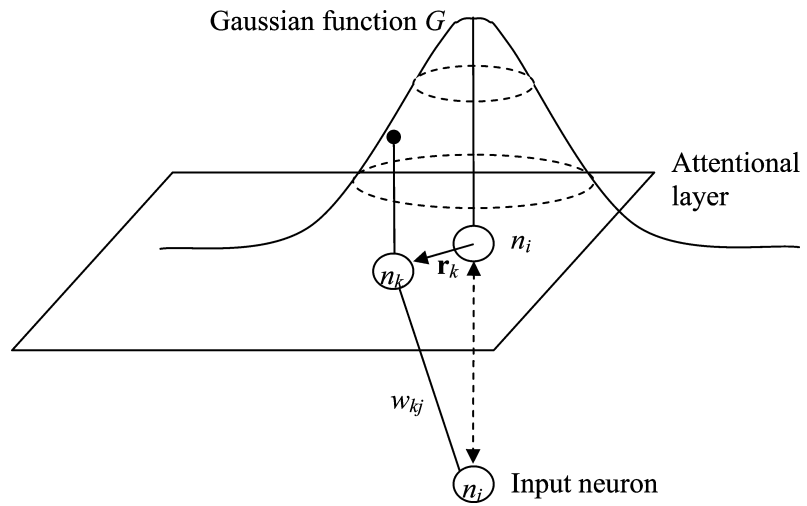

Fig. 6. Weights between input and attentional layers.

Suppose a point stimulus is applied to an input neuron. Next it innervates the neurons in the attentional layer. Rather than equally stimulate, the degrees of innervation associated with attentional neurons are governed by a Gaussian distribution (see Fig. 6). Due to such a distribution, the point stimulus is spread out before arriving at the attentional layer. Recall that the lateral interactions among attentional neurons are dictated by a Mexican-hat function for which the activations of attentional neurons tend to be clustered. The spreading and clustering mechanisms of the STA neural network are in a sense analogous to the morphological operations of dilation and erosion, respectively. The hybrid of the two mechanisms increases the ability of the system to tolerate imperfect input data.

The input stimuli of the STA neural network are the outputs of the sensory analyzers, shown in Fig. 4B. Fig. 4C shows the corresponding attention map of the STA neural network. The spreading and clustering mechanisms make the focus of attention obvious, although the input stimuli are faint. Once the focus of attention is developed, the following subsystems will pay attention to only the area of interest and ignore the rest of the input pattern. It can significantly reduce the search time of the following subsystems.

\subsubsection{Categorical feature extraction}

The purpose of road sign classification is to reduce the search space and increase the recognition rate. Categorical features utilized to partition road signs into groups should represent common characteristics of the groups, not the specific ones of road signs. For example, the red circle regulatory signs may belong to one group, and the blue rectangular information signs may belong to another. The color and shape features of each group should be extracted for classification.

First, create an edge image $E$ from the original stimuli $O$, the input $Z$ of perceptual analyzer, and the attention map $M$ of STA neural network. For each pixel $(x, y)$, $E(x, y)$ is calculated by 


$$
E(x, y)= \begin{cases}\left|E^{\prime}(x, y)\right| & \text { if } M(x, y)>0 \text { and } Z(x, y)>\Gamma_{c}, \\ 0 & \text { otherwise, }\end{cases}
$$

where $\left|E^{\prime}(x, y)\right|$ is the absolute edge magnitude of pixel $(x, y)$, and $\Gamma_{c}$ is the similarity threshold to determinate whether the color of pixel $(x, y)$ has a road sign color. Here, image $E(x, y)$ represents only the edge information of pixels which may belong to the road signs, and the other edge information is filtered by this equation.

Second, by combining the color and edge information, we can locate the candidate positions of road signs. Let $Q$ contain the candidate positions of road signs. For each position $(x, y)$,

$$
Q(x, y)= \begin{cases}1 & \text { if } E(x, y)>0 \text { and } Z(x, y)>0 \\ 0 & \text { otherwise }\end{cases}
$$

As mentioned above, road signs are always installed either beside roads or above lanes, which is where people expect to find them. A pre-attention map is used for modeling the expectation of the human brain. In the pre-attention map, the places where we expect road signs to be located have stronger stimuli than others. Now, we combine this prior information in $Q$ :

$$
\begin{aligned}
& P(x, y)= \begin{cases}1 & \text { if } M^{*}(x, y)>0 \text { and } Q(x, y)=1, \\
0 & \text { otherwise, }\end{cases} \\
& M^{*}(x, y)=\min \left(M(x, y), M^{p}(x, y)\right),
\end{aligned}
$$

where $M^{p}(x, y)$ is the pre-attention stimulus of pixel $(x, y)$. Fig. 7 gives an example to illustrate the effect of the pre-attention map. The original input stimulus is

A

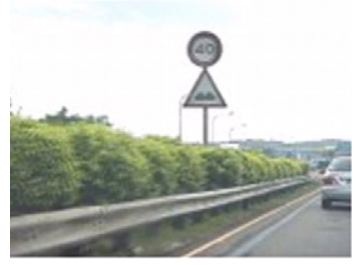

D

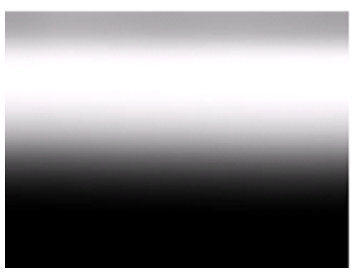

B

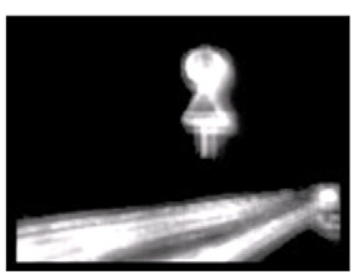

$\mathbf{E}$

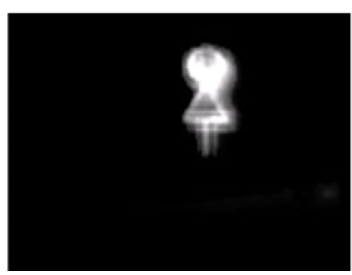

C

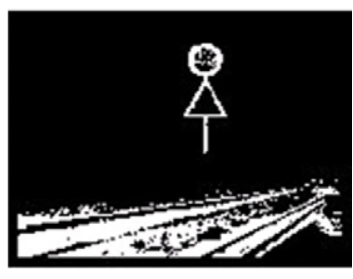

$\mathbf{F}$

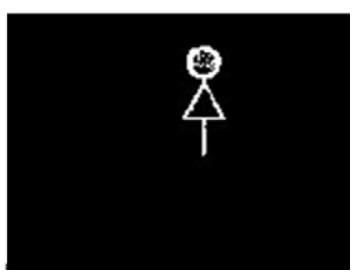

Fig. 7. Experimental results with and without pre-attention. (A) Original image. (B) Attention map without pre-attention. (C) Regions containing road sign candidates without pre-attention. (D) Preattention map. (E) Attention map with pre-attention. (F) Regions containing road sign candidates with pre-attention. 
shown in Fig. 7A, and the pre-attention map is presented in Fig. 7D. Figs. 7B and E show the attention maps, $M$ and $M^{*}$, respectively. Compared with $M$, the attention map given by $M^{*}$ is more concentrated on the road signs, and noise near the ground is filtered out. Finally, patterns $Q$ and $P$ are shown in Figs. 7C and F, respectively.

Next, instead of the traditional method of connected components, we use the complemental connected components technique to detect the road signs. The connected components of the background of $P$ are labeled first and called the background components. Based on these labeled components, only the connected components of the foreground, called the foreground components, surrounding them are of concern. Road signs are always composed of color rims with black/ white interiors. The background components contain the interior parts of road signs, and the foregrounds contain the rims of the road signs. Therefore, road signs are located at the place where foreground components surround the background ones. Fig. 8 shows the difference between these two methods. The traditional method (Fig. 8A) cannot divide the two connected road signs, while the complemental method (Fig. 8B) can. In summary, road sign detection is accomplished by these steps.

After the road sign detection stage, the perceptual analyzer extracts the categorical features input to the conceptual analyzer. The categorical features indicate the color horizontal projection of the road signs. First, the road sign regions are normalized to $N \times M$ pixels. Suppose there are $q$ different road sign colors (e.g., red, green, blue, orange, and yellow), then the categorical feature vector $\mathbf{F}_{c}=\left(\mathbf{f}_{1}, \mathbf{f}_{2}, \ldots, \mathbf{f}_{q}\right)$, where $f_{i}=\left(f_{i 1}, f_{i 2}, \ldots, f_{i N}\right), i=1,2, \ldots, q$, and $f_{i j}$ is the number of pixels which have the $i$ th color in the $j$ th row.

In the color horizontal projection, all gray pixels, including black and white, are treated as the same color to eliminate the individual difference among road signs of the same class. For example, the two warning signs shown in Fig. 9 belong to the same class (red triangular class). The only way to distinguish them is by the distribution of black and white pixels. If these two kinds of pixels are treated as the same color, then the color horizontal projection of the two warning signs should be similar.
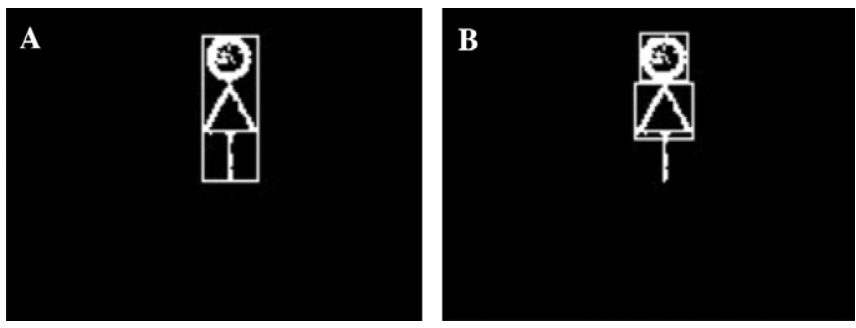

Fig. 8. Results of different connected components methods. (A) Traditional connected components method. (B) Complemental connected component method. 

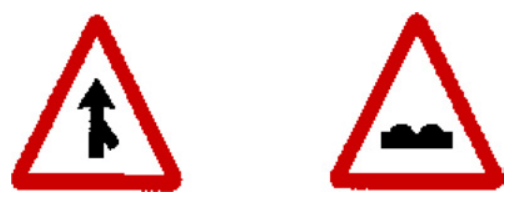

Fig. 9. Two warning signs.

\subsection{Conceptual component}

The conceptual component is divided into two modules: a category module and an object module. The former uses a CART neural network to determine the category of the input stimuli. The later uses a CHAM neural network to recognize an object in a determined category of objects.

\subsubsection{Category module}

CART neural network. The categorical feature extracted in the perceptual component serves as a supraliminal feature to be fed into the CART network in the conceptual component. The CART network is actually an ART2 neural network [12] with a CLTM. Fig. 10 depicts the architecture of the ART2 neural network.

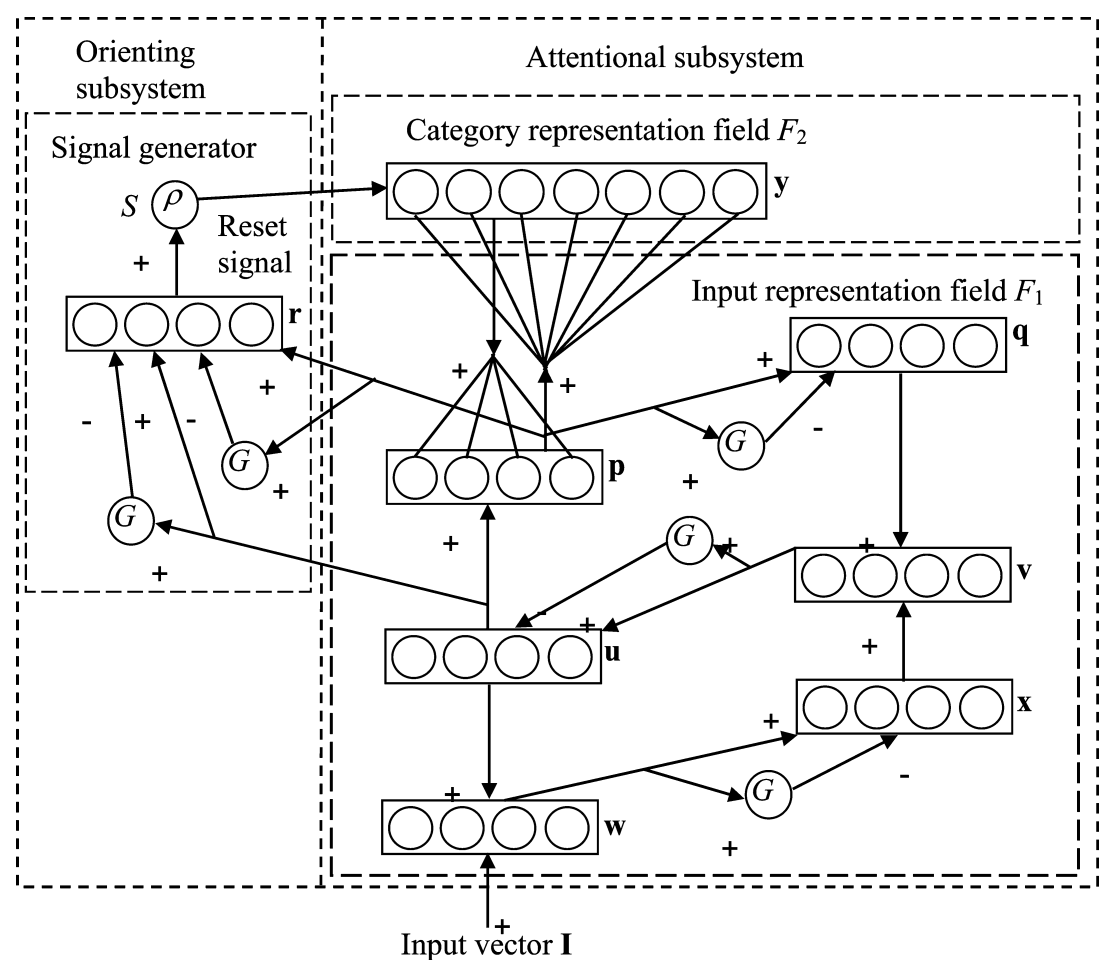

Fig. 10. ART2 neural network. 
The LTM of the neural network is embedded in the bidirectional links between the category (the $F_{2}$ layer) and the input (the $F_{1}$ layer) representation fields in the attentional subsystem. Rather than being fixed, the links and the associated components are reconfigurable. In our proposed computational model, several systems (e.g., road sign, obstacle, traffic condition, and environmental change detection systems) share a common storage, referred to as the episodic memory. The subliminal features of the system under consideration will be loaded from the episodic memory into the LTM of the neural network. The links and associated components of the LTM have to be configured accordingly.

The supraliminal feature (the input categorical feature) is fed into the $F_{1}$ layer of the neural network. Its output is then used as a reference for recalling a subliminal feature from the LTM. Next, the selected subliminal feature is matched with the supraliminal feature in the $F_{1}$ layer. The orienting subsystem of the network decides to accept or reject the subliminal feature based on its degree of match with the supraliminal feature. If the subliminal feature is accepted, the class represented by the subliminal feature is regarded as that of the input supraliminal feature. Supervised learning is then performed to update the subliminal feature in the LTM under the guidance of the supraliminal feature. On the other hand, if the subliminal feature is rejected and there are still unselected subliminal features available in the LTM, the next best subliminal feature is selected and the above process repeats. However, if no subliminal feature matches the supraliminal feature, it is memorized as a new subliminal feature and stored in the LTM through an unsupervised learning process.

The episodic memory in our system has to be refreshed with the content of the LTM of the CART network when the road sign recognition system is terminated. The latest subliminal features are thus preserved for later uses. Furthermore, the CART network can be utilized by the other systems.

Fig. 11 shows a classification result of the CART2 network. We scan sixteen road sign images and extract their categorical features to train and test our system. Sixteen categorical features of road signs are applied to the CART network. These features are classified into eight classes so that similar features will be classified into the same class. Instead of presenting the features, their corresponding road signs are illustrated in Fig. 11. This result is memorized as the learned experience and used to classify the subsequent input features.

Object feature extraction. After a road sign has been classified into some specific class, the object features, which differ from categorical features, should focus on the distinctions of the road signs of the same class. For example, the road signs shown in row of Fig. 12A are all regulatory signs, having the same red rim and circular shape. However, based on the distributions of white and black pixels, these signs represent various meanings, such as no U-turn, left-turn, or right-turn. The distributions of white and black pixels are extracted as the object features for the road sign recognition.

Instead of global thresholding, locally variable thresholding [27] is used to binarize the road sign pattern. In this variable thresholding technique, the input image is first divided into non-overlapping blocks. For each block, a gray-level histogram is computed and a least-squares fit of a bimodal Gaussian mixture to the histogram 
$\mathbf{A}$
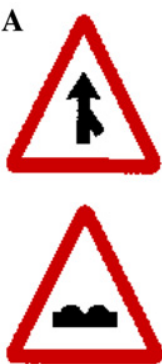

$\mathbf{E}$

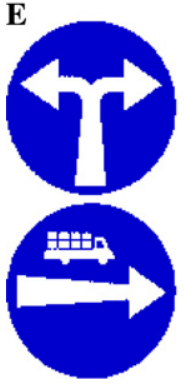

B
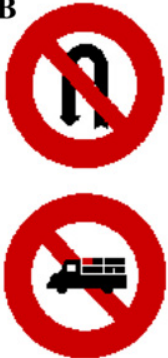

F
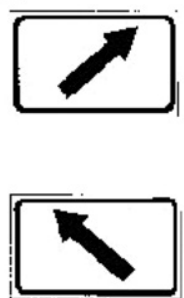

C
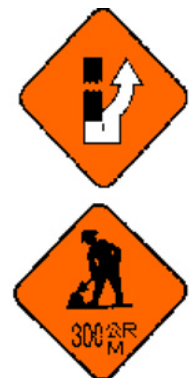

G

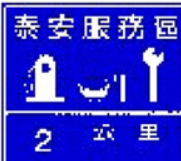

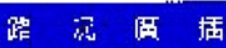

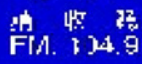

D

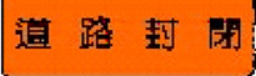

道锱轮 工
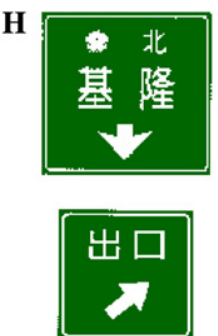

Fig. 11. Classification result of CART2 neural network. (A) Warning signs. (B) Regulatory signs. (C) Construction signs. (D) Construction signs. (E) Guide signs. (F) Guide signs in highway. (G) Information signs in highway. (H) Guide signs in highway.

A
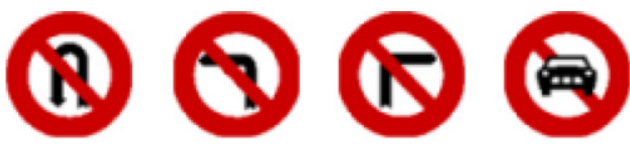

B
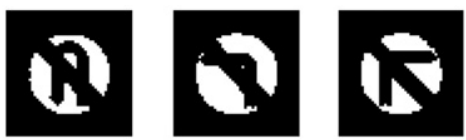

C
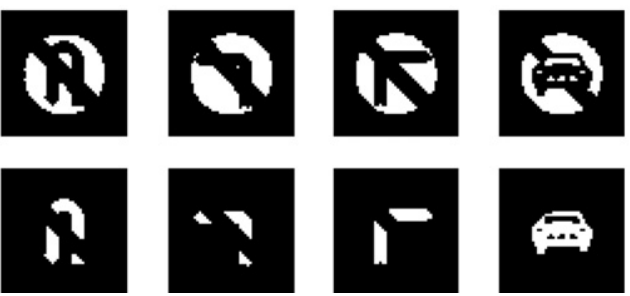
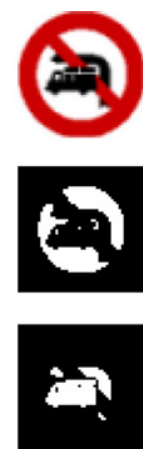

Fig. 12. Some examples of regulatory signs and their extracted object features. (A) The original road signs. (B) Their white object features. (C) Their black object features.

is performed. A threshold value is then determined as the intensity value corresponding to the deepest valley of the fitted curve. Based on these values, a homogenous region test is conducted for the block under consideration. If the block region is regarded as a homogenous area, the determined threshold value is ignored. Finally, from the threshold values of blocks, the dense threshold values of image pixel are computed using bilinear interpolation. This method is particularly useful for dealing 
with the shadowing problem; for example, to threshold the road signs partially shaded by buildings or trees (especially with their leaves swinging in the breeze). After thresholding, the road signs are divided into two parts, black and white, shown in rows of Figs. 12B and C. Both parts are the object features which are fed into the object module for road sign recognition.

\subsubsection{Object module}

Associative memory [12] is one of the primary functions of the human brain. For example, humans can easily associate the face of a friend with that friend's name, or a name with a telephone number. Associative memory can be thought of as one kind of neural network mapping, which is able to compute some functional relationship between its inputs and outputs. Heteroassociative memory is one form of associative memory whose input vector and output vector can be selected freely and independently of each other. Suppose there are $L$ pairs of vectors, $\left\{\left(\mathbf{z}_{1}, \mathbf{m}_{1}\right),\left(\mathbf{z}_{2}, \mathbf{m}_{2}\right), \ldots\right.$, $\left.\left(\mathbf{z}_{L}, \mathbf{m}_{L}\right)\right\}$, with $\mathbf{z}_{i} \in \mathbf{R}^{m}$, and $\mathbf{m}_{i} \in \mathbf{R}^{n}$. A mapping, $\Phi$, of $\mathbf{z}$ to $\mathbf{m}$ can be defined as $\Phi\left(\mathbf{z}_{i}\right)=\mathbf{m}_{i}$. Heteroassociative memory associates non-equal pairs of vectors $\left(\mathbf{z}_{i} \neq \mathbf{m}_{i}\right)$. Similarly, a recognition system which accepts feature input vectors and outputs the recognition results can be thought of as a mapping system whose input and output vectors are different. Moreover, heteroassociative memory is able to tolerate partially occluding and noise interference. Thus, CHAM is selected to recognize objects in our system.

The CHAM neural network, shown in Fig. 13, is structured as a two-layer network with one input layer and one output layer. The output layer is a winnertake-all competitive layer. In the input layer, neurons are arranged into a 2D array, and there are no within-layer synaptic links among these neurons. However, neurons on the output layer are interconnected to one another by within-layer connections and are fully connected to the output neurons by between-layer connections.

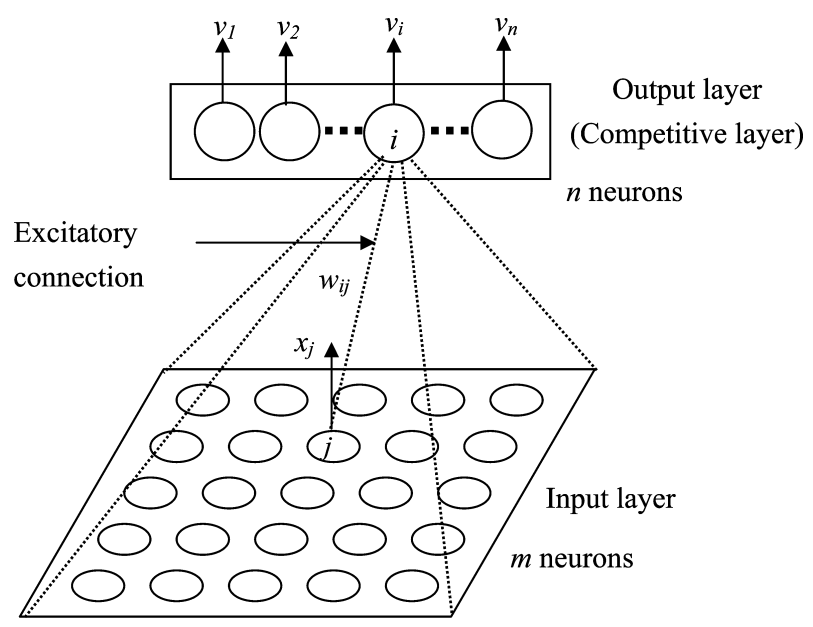

Fig. 13. CHAM neural network. 
Between-layer connections are always excitatory, while within-layer connections are almost always inhibitory.

Suppose that the input layer of the neural network contains $m$ neurons and the output layer contains $n$ neurons. Let $w_{i j}$ denote the weight representing the strength of the link between output neuron $i$ and input neuron $j$. The weight vector of neuron $i$ is written as $\mathbf{w}_{i}=\left(w_{i 1}, w_{i 2}, \ldots, w_{i m}\right)$. The net input to neuron $i$ on the competitive layer due to innervation $\mathbf{z}$ is computed from

$$
\text { net }_{i}=\mathbf{w}_{i} \cdot \mathbf{z}=\sum_{j=1}^{m} w_{i j} z_{j} .
$$

The neurons on the competitive layer will compete among themselves, and the neuron whose weight vector most closely matches the input vector wins. In other words, the winner is the neuron having the largest activation. Let $n_{c}$ be the winner after the competition, then

$$
n_{c}=\arg \left(\max _{i}\left(\text { net }_{i}\right)\right) .
$$

Finally, the one winner on the competitive layer outputs a one while the rest output zeros.

$$
v_{i}= \begin{cases}1 & \text { if } i=n_{c} \\ 0 & \text { otherwise }\end{cases}
$$

Let $\left\{\Theta_{1}, \Theta_{2}, \ldots, \Theta_{p}\right\}$ be the set of object feature patterns stored in the CHAM, where $\Theta_{i}=\left(\Theta_{i b}, \Theta_{i w}\right)^{T}$, and $i=1,2, \ldots, p$. Patterns $\Theta_{i b}$ and $\Theta_{i w}$ represent the black and white feature patterns, respectively, of the $i$ th road sign. If an input object feature pattern, $\Theta$, is fed into the CHAM, then the output class carried out by Eqs. (1) and (2) corresponds to

$$
\Lambda=\arg \left(\min _{i=1, \ldots, p}\left\|\Theta-\Theta_{i}\right\|\right)
$$

where $\left\|\Theta-\Theta_{i}\right\|$ is the distance between $\Theta$ and $\Theta_{i}$.

Fig. 14 shows an example of the regulatory sign recognition. The CHAM is trained by the set of 31 regulatory signs shown in column (A). The white and black object features extracted from the training set are shown in columns (B) and (C), respectively. Three test sets are prepared for the test stage. The first test set, shown in column (D), contains the smoothed images of those signs in column (A) with $5 \times 5$ neighborhood averaging. Their white and black object features extracted from the training set are represented in columns (E) and $(\mathrm{F})$, respectively. The second test set, shown in column $(\mathrm{G})$, is comprised of the regulatory signs corrupted by $20 \%$ uniformly distributed random noise, and the last one, shown in column (J), includes the same signs corrupted by $30 \%$ noise. All 31 smoothed signs and 46 signs with noise are recognized correctly.

Fig. 15 gives another example of regulatory sign recognition. The training set is the same as shown in Fig. 14A but the test patterns (Fig. 15A) are extracted from real images captured by camcorder. Their white and black object features are shown in columns (B) and (C), respectively. Column (D) shows the recogni- 
tion results. Although these road signs are imperfect, they are still recognized by CHAM.

Fig. 16 illustrates an example of warning sign recognition. The CHAM is trained by the set of 20 warning signs shown in column (A). The patterns in columns (B)-(L)

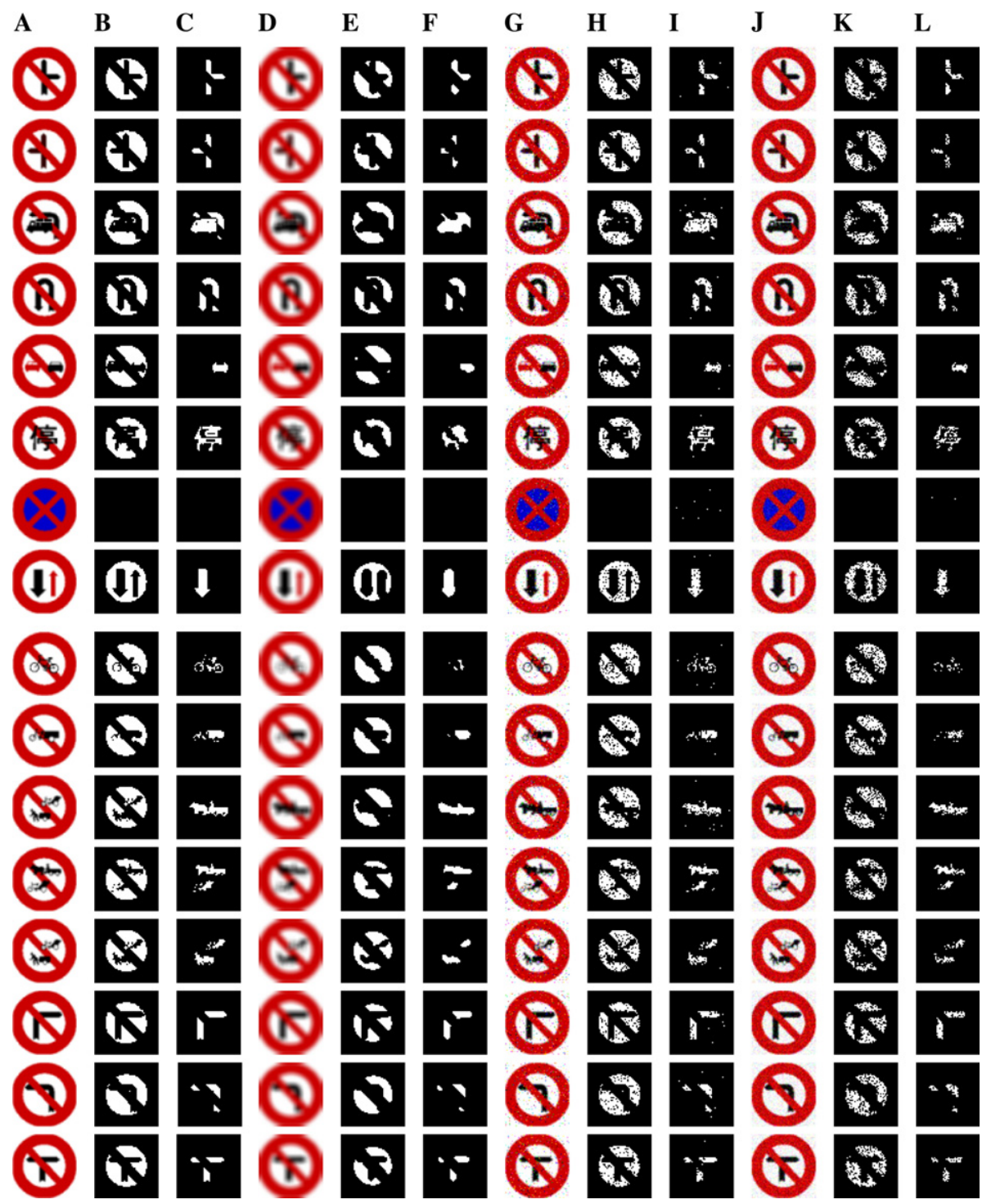

Fig. 14. An experimental result of regulatory signs recognition. (A) Training set of the regulatory signs. (B) White object feature of column (A). (C) Black object feature of column (A). (D) Blurred test set. (E) White object feature of column (D). (F) Black object feature of column (D). (G) Noisy test set. (H) White object feature of column (G). (I) Black object feature of column (G). (J) Noisy test set. (K) White object feature of column (J). (L) Black object feature of column (J). 
are generated in the same way as those in Figs. 14B-L. Columns (D), (G), and (K) are the test sets prepared for the test stage. All 20 smoothed signs and 40 signs with noise are recognized correctly.

These examples show how to recognize road signs in a single image. However, the data input to our system are video sequences. We can collect more information during several successive image frames to make a better decision. As the road signs on the roadside get closer to the vehicle, the images of the road signs projected in the video images continuously increase in size and clarity. While road signs should still

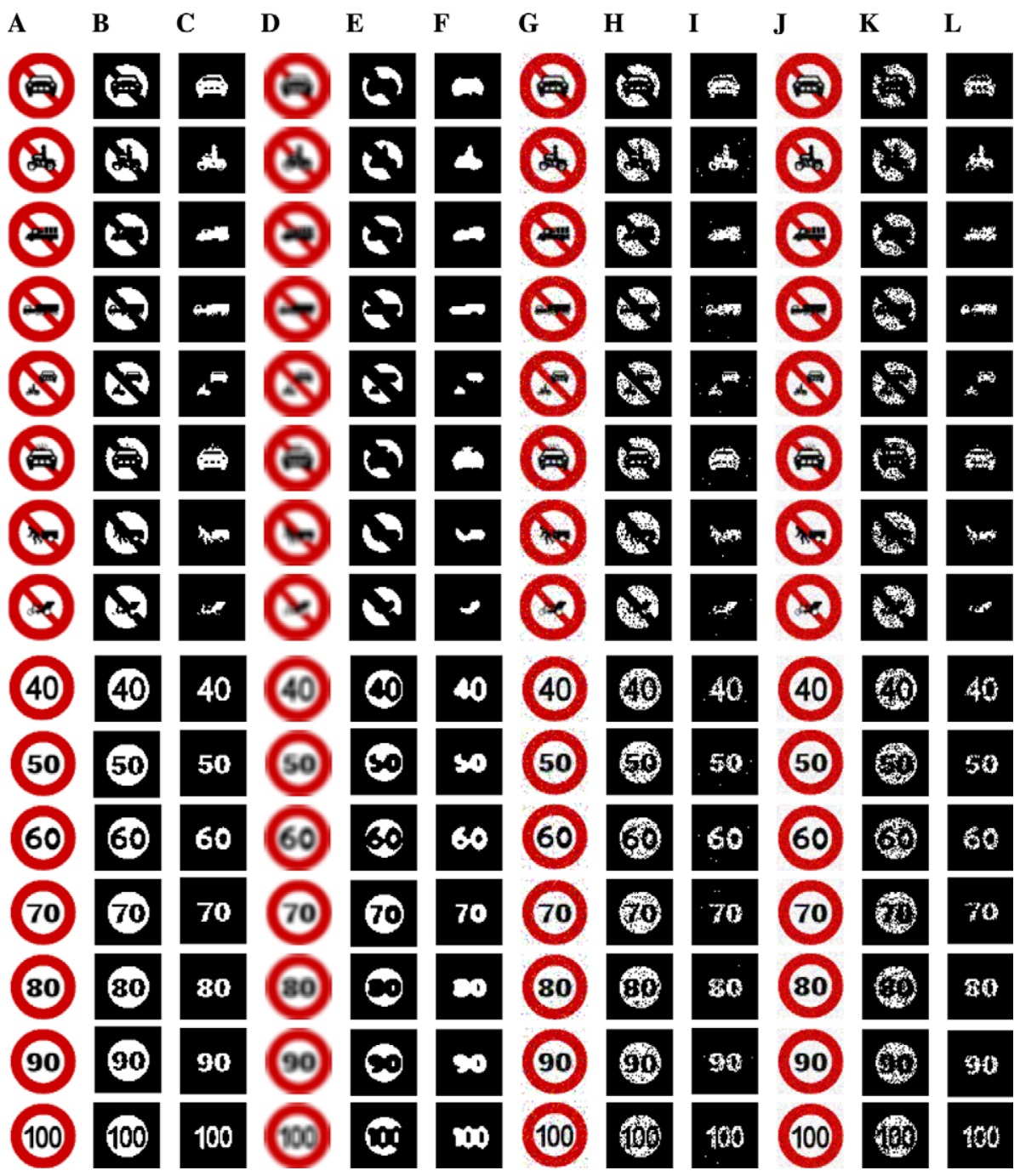

Fig 14. (continued) 


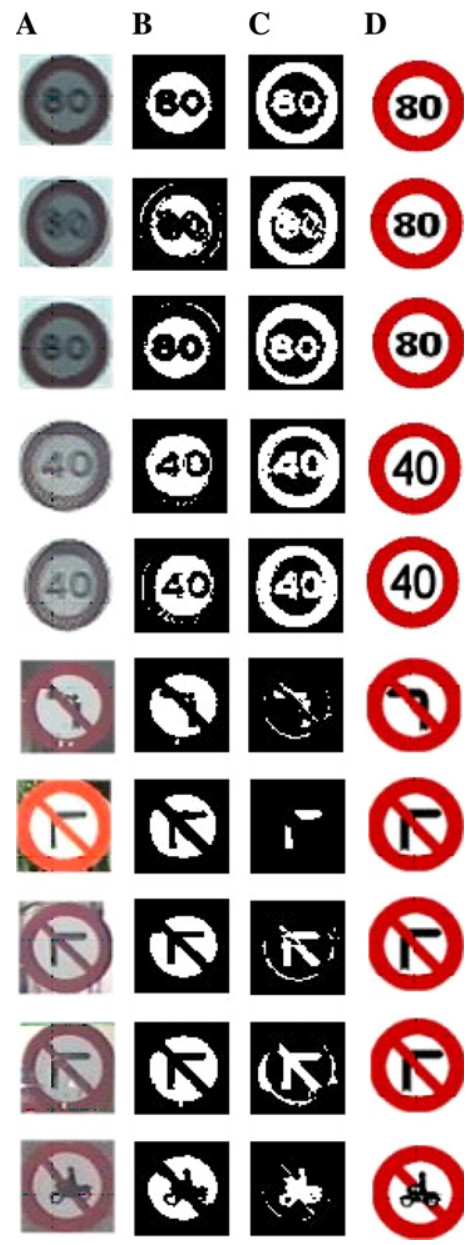

Fig. 15. Recognition results of real road sign patterns. (A) Test images captured by camcorder. (B) Corresponding white object feature of column (A). (C) Corresponding black object feature of column (A). (D) Their recognition results.

be very small at first detection, our system may have difficulty recognizing these small signs. However, such signs still supply valuable information for eliminating impossible candidates of road signs and reducing the search space. The more video images fed into our system, the more information can be used to strengthen our decision.

Suppose our system initially maintains all the candidates for road signs in the LTM of the CHAM network, then after processing some of the video input, the number of candidates, $p$, will be decreased by using the following procedure: 


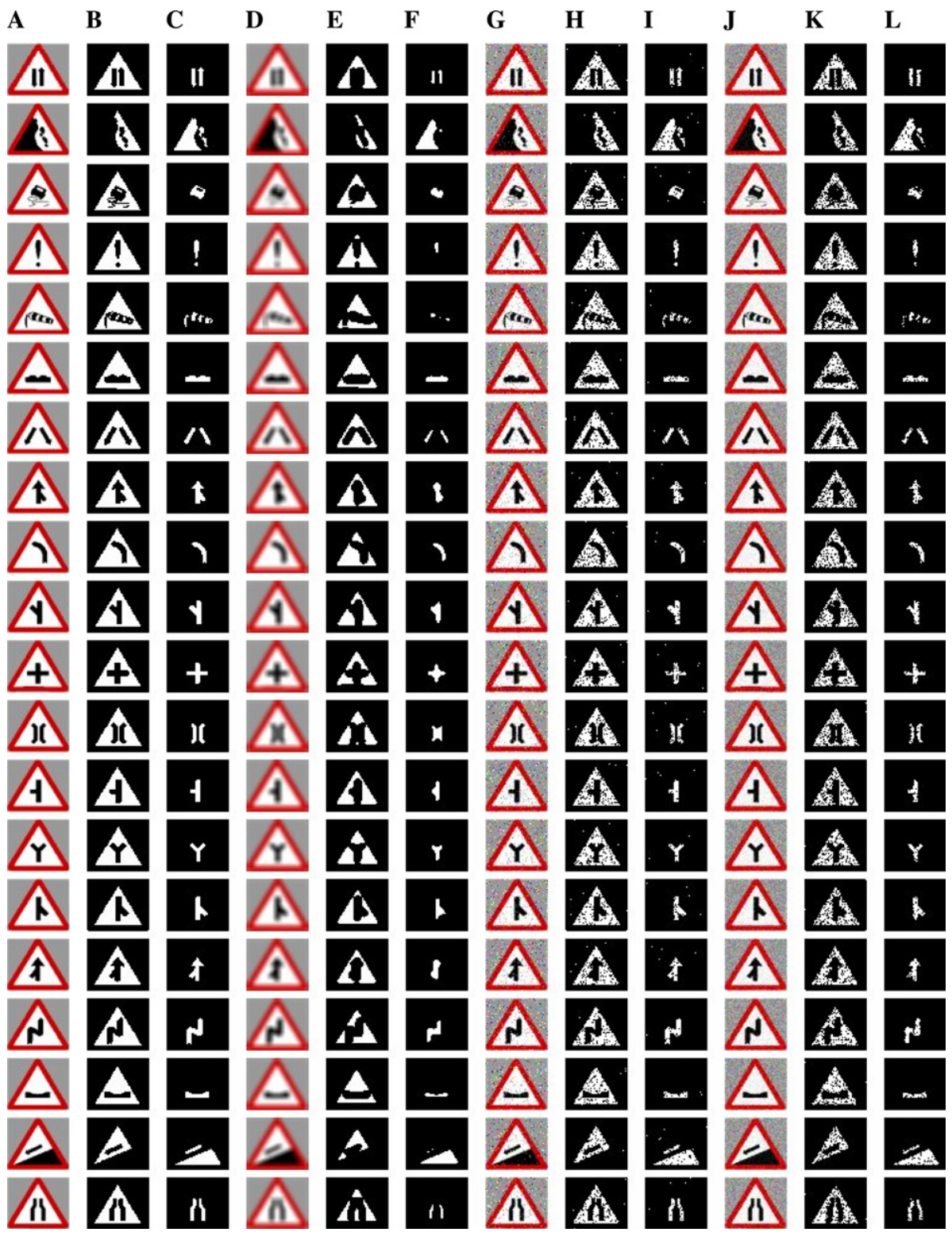

Fig. 16. An experimental result of warning sign recognition. (A) Training set of the regulatory signs. (B) White object feature of column (A). (C) Black object feature of column (A). (D) Blurred test set. (E) White object feature of column (D). (F) Black object feature of column (D). (G) Noisy test set. (H) White object feature of column (G). (I) Black object feature of column (G). (J) Noisy test set. (K) White object feature of column (J). (L) Black object feature of column (J). 
(1) Put all candidates for the road signs in the LTM.

(2) Input the extracted object feature $\Theta$ of an image in a video sequence to the CHAM network.

(3) For each candidate $\Theta_{j}$ in the LTM, apply the following rule: If $\left\|\Theta-\Theta_{j}\right\|>\frac{1}{p} \sum_{i=1}^{p}\left\|\Theta-\Theta_{i}\right\|$, then remove the $j$ th candidate from the LTM, where $j=1,2, \ldots, p$.

(4) Repeat steps (2) and (3) until only one candidate is left, and output the candidate as the recognition result.

This procedure illustrates the recognition process for a road sign. If there are two or more signs in the image sequence, the recognition process is the same except that the contents of the LTM should be modified for the different signs.

\section{Experimental results and discussion}

As mentioned above, the input data to our system were acquired using a video camcorder mounted in the front windshield of a vehicle while driving on freeways. Since the vehicle's speed is high, moving objects appear jerky if the image is read/output about every 1/30s. Fig. 17 shows an example of this situation.

In our experiments, each video sequence was down-sampled to a frame rate of $5 \mathrm{~Hz}$ before being submitted to the system. Furthermore, each $720 \times 480$ pixel input image was reduced to $180 \times 120$ pixels by uniform sub-sampling. It is well known that the advantages of sub-sampling include the reduction of processing time, noise,

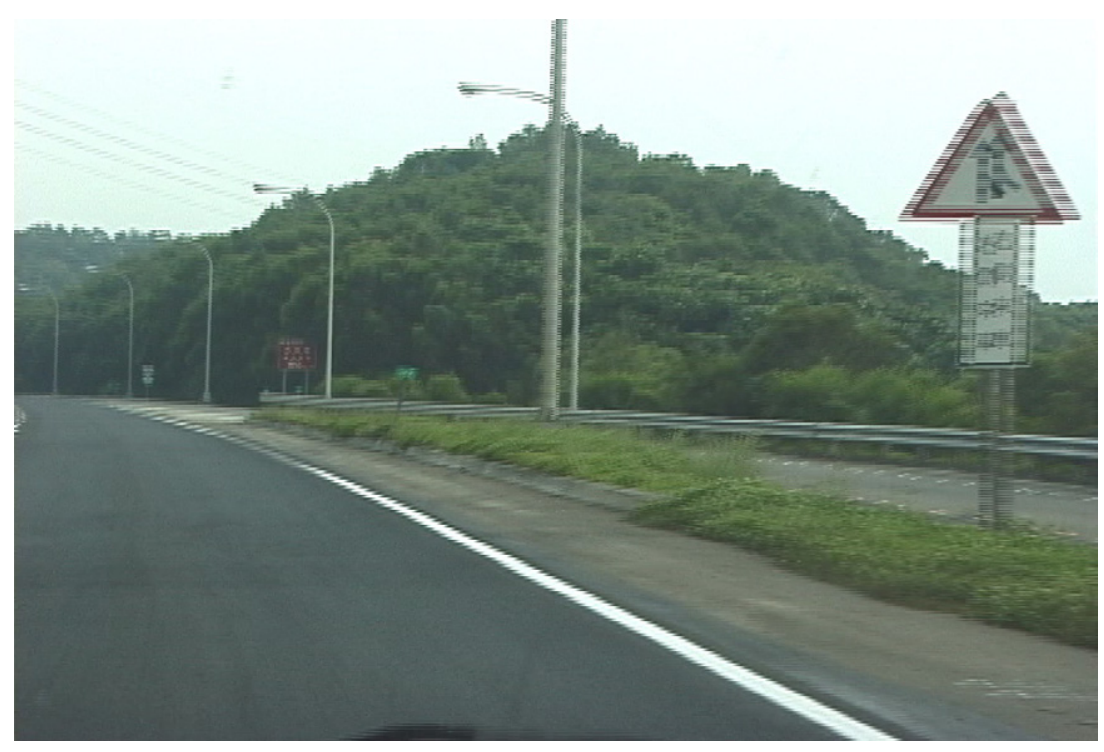

Fig. 17. Original image captured with camcorder. 
$\mathbf{A}$

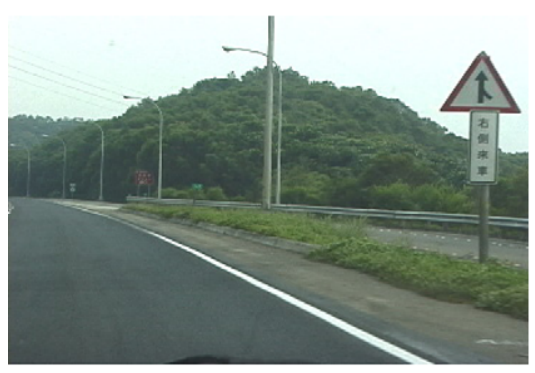

B

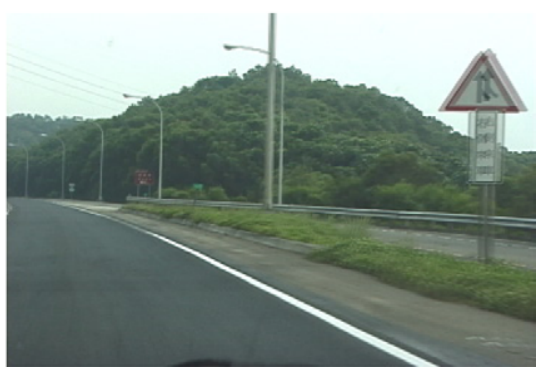

Fig. 18. Results of different sub-sampling methods. (A) Result of uniform sub-sampling method. (B) Result of averaging sub-sampling method.

as well as image instability. However, we want to emphasize that uniform subsampling is better than averaging. In Figs. 18A and B, the different can easily be discerned. These two images are derived from Fig. 17, and only uniform sub-sampling will produce clear images in high-speed situations.

\subsection{Experimental results}

A number of video sequences were collected for experiments with one or two signs included in each sequence. The first example of the experimental results is presented in Fig. 19. It shows only part of a video sequence (the seven images in column (A)). In this sequence, two road signs should be recognized, one is a speed limit sign and the other is a warning sign. The corresponding attention maps of the input images, column (A), are shown in column (B). These attention maps have combined pre-attention information, and thus the focus of attention is concentrated on where the road signs are located. Column (C) represents the detection results of the candidate road signs. We frame these road signs with white boxes. Column (D) shows the contents of the white boxes. They are extracted from the input image and normalized to $60 \times 60$ pixels. The normalized patterns are first input to the CART network for classification and then fed to the CHAM network for recognition. The recognized results, shown in column (E), are output only when one candidate is left in each LTM. In this example, the warning sign is recognized first, and the speed limit sign is recognized three images later.

Another example of the experimental results is presented in Fig. 20, showing only five images of a video sequence (column (A)). This sequence includes two speed limit signs. Column (B) shows the corresponding attention maps for the input images of column (A); and column (C) gives the detection results of the candidate road signs. The extracted and normalized patterns $(60 \times 60$ pixels $)$ for road sign recognition are shown in column (D) and the recognized results in column (E). In this example, the lower speed limit sign is recognized first, and the upper sign is recognized three images later. 
A
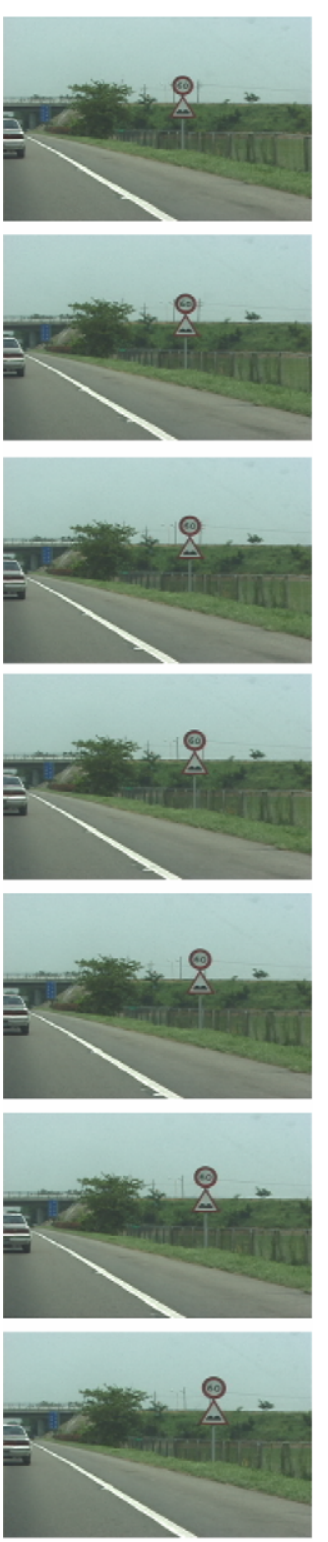

B
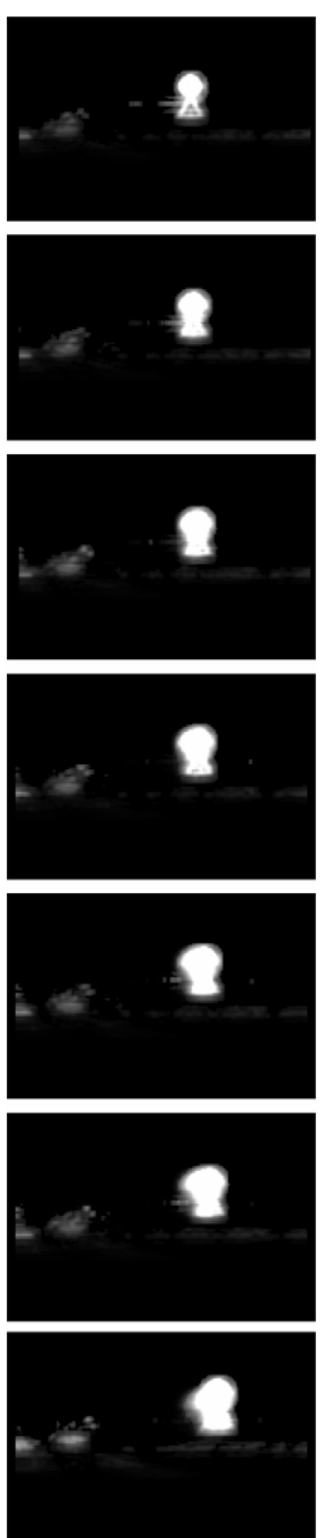

C
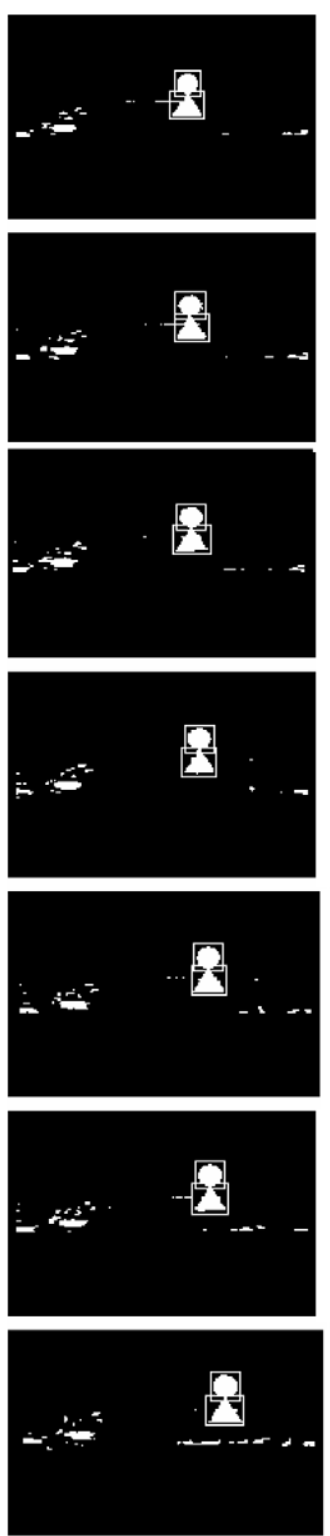

D
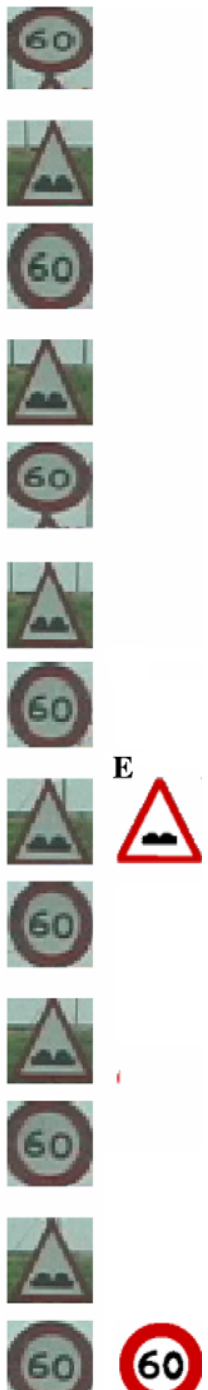

60

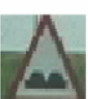

Fig. 19. Result of road sign detection and recognition. (A) The input video sequence. (B) The corresponding attention map. (C) The results of road sign detection. (D) The road signs extracted after category module. (E) The recognition result.

Fig. 21 shows six more examples of experimental results. In each sequence we select only one image to represent the whole sequence in this figure. Columns (A) and (D) are the selected images of the input video sequences, and columns (B) and (E) 
A
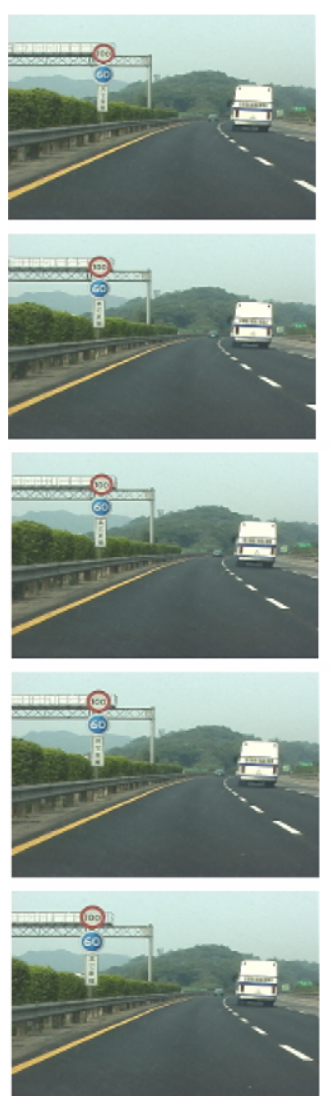

B
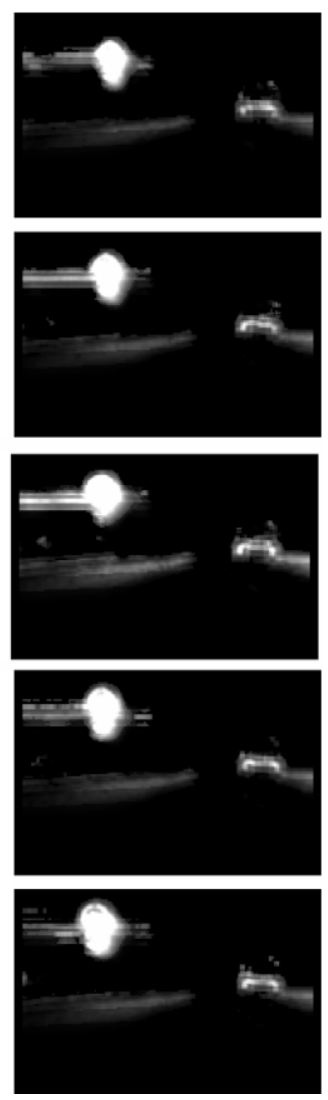

C
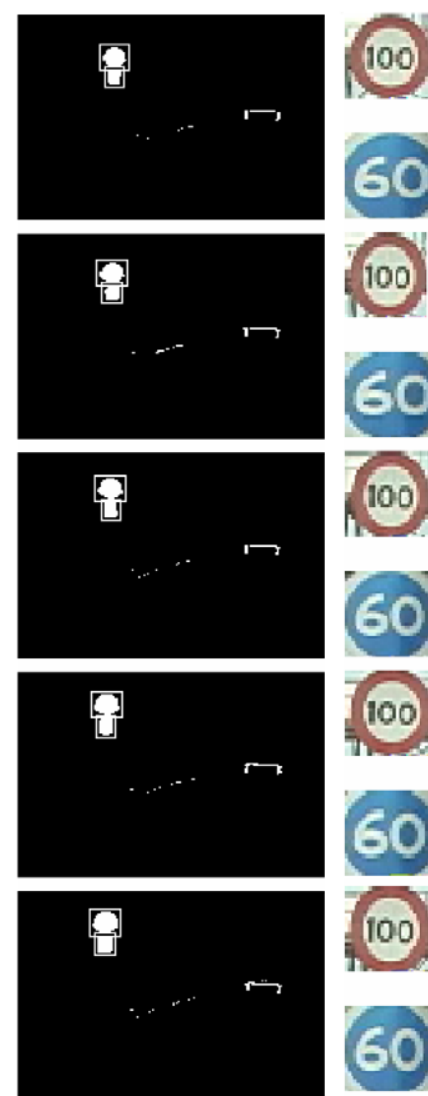

D
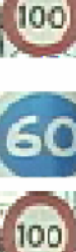

$\mathbf{E}$
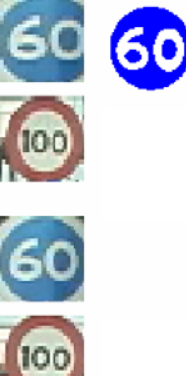

5
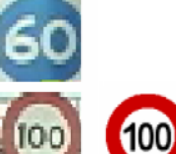

Fig. 20. Another experimental result of road sign detection and recognition. (A) The input video sequence. (B) The corresponding attention map. (C) The results of road sign detection. (D) The road signs extracted after category module. (E) The recognition result.

give their detection results. The recognition results are presented in columns $(\mathrm{C})$ and (F). No matter how many road signs are contained in the sequences, the recognition results are all correct.

Although most road signs in input images are detected at the detection stage, there are some missed detections. Missed detection usually occurs when the road signs are small in the image. These road signs may have no strong rims for detecting correct connected components. Another reason is that the color on the rim of the road signs does not match its original color (e.g., affected by colored lighting). Fig. 22 shows two examples of missed detection. Fig. 22B shows the result of Fig. 22A. The warning sign is not detected, because its rim is not complete so as to separate the interior and exterior of the sign. Figs. 22C and D show another example of missed detection. Since our system integrates the results of several 
A
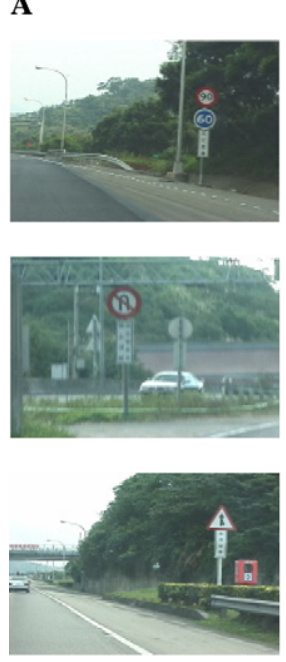

B
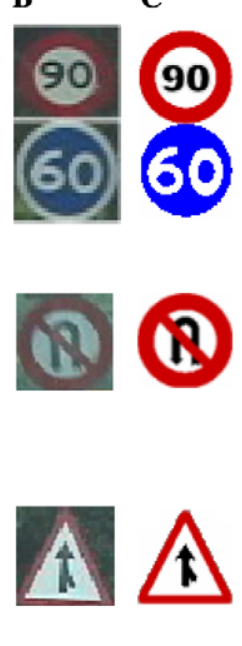

C
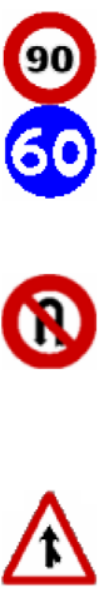

D

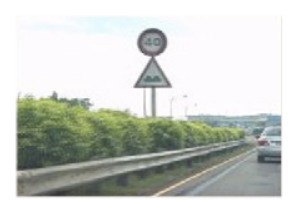

E $\quad \mathbf{F}$
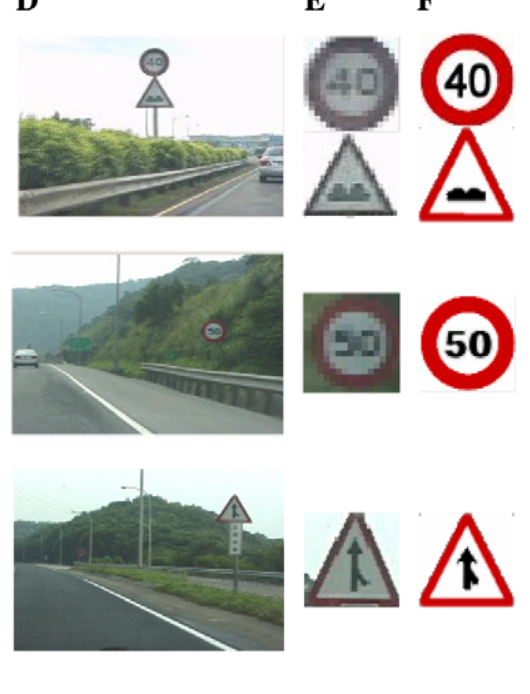

Fig. 21. Some experimental results of road sign detection and recognition. (A,D) are the input video sequences. (B,E) Detection results. (C,F) The recognition results.

A

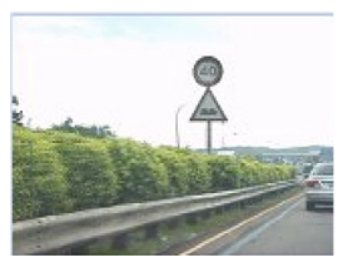

C

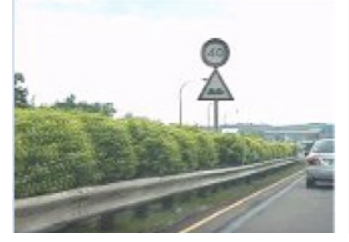

B

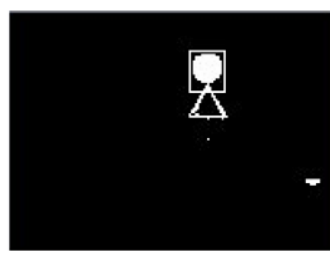

D

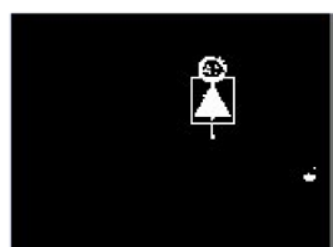

Fig. 22. Two examples of missed detection. (A) The first input image. (B) The road sign detection result of (A). (C) The second input image. (D) The road sign detection result of (C).

successive images to make a decision, a few missed detections in a sequence does not affect the decision making of our system.

If the speed of a vehicle is very high or the road surface is rough, then vehicle and camcorder vibration cannot be avoided, and the quality of input video sequences is reduced. Some patterns extracted from these sequences, shown in Fig. 23A, appear 


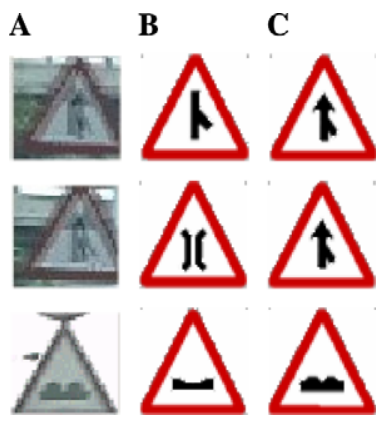

Fig. 23. Three examples of incorrect recognition. (A) The extracted pattern for recognition. (B) Their recognition results. (C) The correct answers.

jerky, although they are detected and classified correctly. However, the recognition of these patterns may be incorrect. Comparing the incorrect answers (Fig. 23B) with the correct ones (Fig. 23C) shows that they are similar but not exactly the same. The incorrect results do not affect the correctness of system's decision since the decision is made by integrating several images.

Twenty-one sequences have been tested in our experiment. Most of the classification results of the CART neural network are correct (99\%), and only 11 frames (1\%) are incorrect. About $85 \%$ of extracted road signs can be recognized correctly in the CHAM neural network. Since our system only outputs a result for each input sequence, this $85 \%$ ratio is enough for our system to recognize road signs correctly.

\subsection{Discussion}

The CART and CHAM networks should be well trained before being tested. As mentioned earlier, the training patterns of CART are shown in Fig. 11. These patterns are first normalized to $60 \times 60$ pixels, and then the categorical feature vectors (color horizontal projection) are extracted to train the CART network. Although the patterns in the training set are man-made, they can be used to classify real patterns captured by the camcorder. In our experiments additional patterns are not need for training the CART network.

For each category stored in the CART networks, a corresponding LTM of the CHAM network should be trained. The weights in these LTMs are recorded in episodic memory, and will be moved to the LTM as they are needed. In our experiments, only three categories in Fig. 11 have been trained. They are the regulatory signs (Fig. 11A), the warning signs (Fig. 11B), and the guide signs (Fig. 11E). The training sets of regulatory and warning signs are shown in Figs. 14A and 16A, respectively. Similar to the training set of the CART network, these patterns can be utilized to recognize the real patterns. In our experiments the lower speed limit signs are included in the set of guide signs. Since the number of training patterns is small, the training set of guide signs is not shown here. 
Our system is able to detect and recognize road signs at night if the street signs are illuminated by headlights or street lights. However, if road signs are in dark or the light is too dim, they may be lost at the detection stage. Our system cannot handle these conditions.

\section{Conclusion and future work}

In this paper, a computational model motivated by human cognitive and recognition processing was presented. Based on the proposed model, a road sign detection and recognition system was developed. The computational model consists of three analyzers: sensory, perceptual, and conceptual. The sensory analyzer extracts both spatial and temporal information from dynamic scenes. The spatial information calls the attention of the perceptual analyzer to interesting objects, whereas the temporal information calls attention to moving objects. The STA neural network involved in the perceptual analyzer realizes both the spatial and temporal attentional mechanisms in one construct. The conceptual analyzer based on CART and CHAM neural networks interprets the focuses of attention formed in the STA network.

The road sign recognition system is not the only subsystem in vision-based DAS. There are several other subsystems which perform road detection, obstacle recognition, and environmental change detection, etc. Developing and integrating these subsystems to collect significant information in driving environments is very important for improving traffic safety. We hope the proposed computational model is helpful for designing various subsystems for functions such as the detection and recognition of lanes, traffic signals, weaving intersections, obstacles, as well as road and weather conditions. Moreover, other applications to event detection and recognition can also be accomplished with this model by extracting different kinds of features.

\section{Acknowledgments}

This work was supported by the National Science Council, Republic of China, under Contract NSC-90-2213-E-003-002. The authors gratefully acknowledge the assistance of Prof. Robert R. Bailey of National Taiwan Normal University for his many helpful suggestions in writing this paper and for editing the English.

\section{References}

[1] Y. Aoyagi, T. Asakura, A study on traffic sign recognition in scene image using genetic algorithms and neural networks, in: Proc. IEEE IECON Int. Conf. on Industrial Electronics, Control, and Instrumentation, Taipei, Taiwan, vol. 3, 1996, pp. 1838-1843.

[2] P. Arnoul, M. Viala, JP. Guerin, M. Mergy, Traffic signs localization for highways inventory from a video camera on board a moving collection van, in: Proc. IEEE Intelligent Vehicles Symposium, Tokyo, Japan, 1996, pp. 141-146. 
[3] M. Bertozzi, A. Broggi, GOLD: a parallel real-time stereo vision system for generic obstacle and lane detection, IEEE Trans. Image Process. 7 (1998) 62-81.

[4] M. Bertozzi, A. Fascioli, A. Broggi, Performance analysis of a low-cost solution to vision-based obstacle detection, in: Proc. IEEE Int. Conf. on Intelligent Transportation Systems, Tokyo, Japan, 1999, pp. 350-355.

[5] R. Chapuis, F. Marmoiton, R. Aufrere, F. Collange, J. P. Derutin, Road detection and vehicles tracking by vision for an On-Board ACC System in the VELAC vehicle, in: Proc. Int. Conf. on Information Fusion, Paris, vol. 2, 2000, pp. 11-18.

[6] A. de la Escalera, L. Moreno, Road traffic sign detection and classification, IEEE Trans. Ind. Electron. 44 (1997) 848-859.

[7] S. Estable, J. Schick, F. Stein, R. Janssen, R. Ott, W. Ritter, Y. J. Zheng, A real-time traffic sign recognition system, in: Proc. of the Intelligent Vehicles Symposium, Paris, 1994, pp. 213-218.

[8] L. Estevez, N. Kehtarnavaz, A real-time histographic approach to road sign recognition, in: Proc. IEEE Southwest Symp. on Image Analysis and Interpretation, San Antonio, Texas, 1996, pp. 95-100.

[9] C.Y. Fang, C.S. Fuh, S.W. Chen, Detection and tracking of road signs, Pattern Recognit. Image Anal. 11 (2001) 304-308.

[10] C.Y. Fang, S.W. Chen, C.S. Fuh, Automatic change detection of driving environments in a visionbased driver assistance system, IEEE Trans. Neural Networks 14 (2003) 646-657.

[11] C.Y. Fang, C.S. Fuh, S.W. Chen, Driving environmental change detection subsystem in a visionbased driver assistance system, in: Proce. IEEE Int. Joint Conf. on Neural Networks, Honolulu, Hawaii, vol. 1, 2002, pp. 246-251.

[12] J.A. Freeman, D.M. Skapura, Neural Networks-Algorithms, Applications, Programming Techniques, Addison-Wesley, Readings, MA, 1992.

[13] D.M. Gavrila, Multi-feature hierarchical template matching using distance transforms, in: Proc. IEEE Int. Conf. Pattern Recognition, Brisbane, Australia, 1998. Available from: <http://www.gavrila.com>.

[14] D. Ghica, S.W. Lu, X. Yuan, Recognition of traffic signs by artificial neural network, in: Proc. IEEE Int. Conf. on Neural Network, Perth, Australia, vol. 3, 1995, pp. 1444-1449.

[15] A. Guiducci, Parametric model of the perspective projection of a road with applications to lane keeping and 3d road reconstruction, Comput. Vis. Image Understand. 73 (1999) 414- 427.

[16] J.A. Janet, M.W. White, T.A. Chase, R.C. Luo, J.C. Sutto, Pattern analysis for autonomous vehicles with the region- and feature-based neural network: global self-localization and traffic sign recognition, in: Proc. IEEE Int. Conf. on Robotics and Automation, Minneapolis, Minnesota, vol. 4, 1996, pp. 3598-3604.

[17] G.Y. Jiang, T.Y. Choi, Y. Zheng, Morphological traffic sign recognitions, in: Proc. Int. Conf. on Signal Processing, Lausanne, Switzerland, vol. 1, 1996, pp. 531-534.

[18] D.S. Kang, A noise tolerant algorithm for the object recognition of warning system, in: Proc. IEEE Int. Symp. on Information Theory, Whistler, Canada, 1995, p. 367.

[19] N. Kehtarnavaz, A. Ahmad, Traffic sign recognition in noisy outdoor scenes, in: Proceedings of the Intelligent Vehicles Symposium, Detroit, 1995, pp. 460-465.

[20] K. Kluge, C. Thorpe, The YARF system for vision-based road following, J. Math. Comput. Modell. 22 (1995) 213-233.

[21] J. Konorski, Integrative Activity of the Brain, University of Chicago Press, Chicago, 1967.

[22] H.S. Lai, H.C. Yung, Lane detection by orientation and length discrimination, IEEE Trans. Syst. Man Cybernet.-Part B: Cybernet. 30 (2000) 539-548.

[23] M. Lalonde, Y. Li, Detection of Road Signs Using Color Indexing, Technical Report CRIM-IT-95/ 12-49, Centre de Recherche Informatique de Montreal. Available from: <http://www.crim.ca/sbc/ english/cime/> publications.html, 1995.

[24] W. Li, X. Jiang, Y. Wang, Road recognition for vision navigation of an autonomous vehicle by fuzzy reasoning, Fuzzy Sets Syst. 93 (1998) 275-280.

[25] S.W. Lu, Recognition of traffic signs using a multilayer neural network, in: Proc. Canadian Conf. on Electrical and Computer Engineering, Halifax, Nova Scotia, vol. 2, 1994, pp. 833-834.

[26] C. Martindale, Cognitive Psychology -A Neural-Network Approach, Brooks/Cole, Pacific Grove California, 1991. 
[27] Y. Nakcogawa, A. Rosenfeld, Some experiments of variable threshold, Pattern Recognit. 11 (1979) 191-204.

[28] D. Nair, J.K. Aggarwal, Moving obstacle detection from a navigating robot, IEEE Trans. Robot. Automat. 14 (1998) 404-416.

[29] L. Pacheco, J. Batlle, X. Cufi, A new approach to real time traffic sign recognition based on colour information, in: Proceedings of the Intelligent Vehicles Symposium, Paris, 1994, pp. 339-344.

[30] G. Piccioli, E.D. Micheli, P. Parodi, M. Campani, A robust method for road sign detection and recognition, Image Vis. Comput. 14 (1996) 209-223.

[31] L. Priese, J. Kileber, R. Lakmann, V. Rehrmann, R. Schian, New results on traffic sign recognition, in: Proceedings of the Intelligent Vehicles Symposium, Paris, 1994, pp. 249-254.

[32] W. Ritter, F. Stein, R. Janssen, Traffic sign recognition using colour information, J. Math. Comput. Modell. 22 (1995) 149-161.

[33] G. Sharma, H.J. Trussell, Digital color imaging, IEEE Trans. Image Process. 6 (1997) 901-932.

[34] H.D. Tagare, K. Toyama, J.G. Wang, A maximum-likelihood strategy for directing attention during visual search, IEEE Trans. Pattern Anal. Mach. Intell. 23 (2001) 490-500.

[35] M. Yamada, K. Ueda, I. Horiba, N. Sugie, Discrimination of the road condition toward understanding of vehicle driving environments, in: Proc. IEEE Int. Conf. on Intelligent Transportation Systems, Tokyo, Japan, 1999, pp. 20-24.

[36] Y. J. Zheng, W. Ritter, R. Janssen, An adaptive system for traffic sign recognition, in: Proceedings of the Intelligent Vehicles Symposium, Paris, 1994, pp. 165-170. 